Homology, Homotopy and Applications, vol. 7(3), 2005, pp.11-36

\title{
ADDITIVITY OF EULER CHARACTERISTICS IN RELATIVE ALGEBRAIC $K$-GROUPS
}

\author{
MANUEL BREUNING AND DAVID BURNS
}

(communicated by J.F. Jardine)

\begin{abstract}
We describe a criterion for a natural Euler characteristic that takes values in a relative algebraic $K_{0}$-group to be additive in distinguished triangles. As preliminary steps we prove several results about determinant functors, in particular concerning the comparison of the determinant of a complex to the determinant of its cohomology.
\end{abstract}

Dedicated to Victor Snaith on the occasion of his 60-th birthday.

\section{Introduction}

Let $\Lambda$ be a ring and $P$ a bounded complex of finitely generated projective $\Lambda$ modules. The Euler characteristic $\chi(P)$ of $P$ is defined to be the alternating sum $\sum_{i \in \mathbb{Z}}(-1)^{i}\left(P^{i}\right)$ in the Grothendieck group $K_{0}(\Lambda)$. An immediate consequence of this definition is that for any short exact sequence $0 \rightarrow P \rightarrow Q \rightarrow R \rightarrow 0$ of such complexes there is an identity

$$
\chi(Q)=\chi(P)+\chi(R) .
$$

In arithmetic algebraic geometry a complex $P$ of $\Lambda$-modules often comes equipped with a trivialisation $t$ over some ring extension $\Sigma$ of $\Lambda$, that is some additional data on the cohomology of the complex $\Sigma \otimes_{\Lambda} P$. In this situation one can construct an Euler characteristic $\chi(P, t)$ in the relative algebraic $K$-group $K_{0}(\Lambda, \Sigma)$ which is a preimage of $\chi(P)$ under the natural homomorphism $K_{0}(\Lambda, \Sigma) \rightarrow K_{0}(\Lambda)$. In this paper we describe a criterion for a short exact sequence $0 \rightarrow P \rightarrow Q \rightarrow$ $R \rightarrow 0$ of trivialised complexes (and more generally for a distinguished triangle of such complexes) which, when satisfied, ensures additivity of the associated Euler characteristics, that is

$$
\chi\left(Q, t_{Q}\right)=\chi\left(P, t_{P}\right)+\chi\left(R, t_{R}\right) .
$$

The first author was partially supported by the Gottlieb Daimler- and Karl Benz-Foundation and by EPSRC grant GR/S91772/01.

Received October 26, 2004, revised February 11, 2005; published on November 12, 2005.

2000 Mathematics Subject Classification: 18E10, 18E30, 18D10, 16E20.

Key words and phrases: Determinant functors, Euler characteristics, relative algebraic $K$-groups. (C) 2005, Manuel Breuning and David Burns. Permission to copy for private use granted. 
Our criterion is of a purely cohomological nature in that it involves the trivialisations and the long exact cohomology sequence but not the complexes themselves. This feature ensures that our result is relevant to several recent constructions in arithmetic algebraic geometry involving (trivialised) perfect complexes which are only well-defined up to quasi-isomorphism. In particular, in a future manuscript we will apply our criterion to certain distinguished triangles that arise in the context of the constructions of [1] and [2]. In addition, recent work of Fukaya and Kato [7] suggests that applications of our result may well arise naturally in non-commutative Iwasawa theory (in particular, in the context of elliptic curves with supersingular reduction for which the relevant Selmer groups are not torsion modules over the appropriate Iwasawa algebra).

As in [4], our approach to Euler characteristics uses determinant functors on categories of bounded complexes with values in categories of virtual objects. In the first half of this paper we prove various properties of very general determinant functors on categories of bounded complexes which may themselves be of independent interest. We start with a summary of some basic notions in $\S 2$ and then discuss the comparison of the determinant of a complex to the determinant of its cohomology in $§ 3$. The key result here is a description of how the isomorphism of determinants associated to a short exact sequence of complexes as above is reflected on the level of cohomology. Section 4 consists of various results which express the determinant of a complex in terms of the determinants of its even and odd degree parts.

The second half of this paper then deals with Euler characteristics. In $\S 5$ we specialise the preceding results to determinant functors with values in certain categories of virtual objects, which allows us to define the Euler characteristic $\chi(P, t)$, establish some of its basic properties and prove the key additivity result. The constructions and results of $\S 5$ are valid whenever $\Sigma$ is both noetherian and regular, but if one assumes that $\Sigma$ is semisimple, then they can be stated in a much more explicit fashion (and, in particular, without reference to virtual objects). This is the content of $\S 6$ where we also establish the relation of our Euler characteristics to the 'refined Euler characteristics' defined by the second named author in [3]. We remark that such a comparison has already been sketched (albeit slightly erroneously - see Remark 6.5) in [4, Remark 4] but no details have appeared before now.

\section{Basics}

Let $\mathcal{P}$ be a commutative Picard category, that is a groupoid $\mathcal{P}$ with a product $\otimes: \mathcal{P} \times \mathcal{P} \rightarrow \mathcal{P}$ which satisfies compatible associativity, commutativity and unit constraints, and for which every object in $\mathcal{P}$ is invertible. We refer the reader to [8, Appendix A] for more details on Picard categories and to [11, Chapitre I] for a thorough study of tensor categories in general. It will be convenient to fix a unit $1_{\mathcal{P}}$ in $\mathcal{P}$, that is an object $1_{\mathcal{P}}$ with an isomorphism $1_{\mathcal{P}} \rightarrow 1_{\mathcal{P}} \otimes 1_{\mathcal{P}}$. Recall that units in a Picard category are unique up to unique isomorphism. In this paper we will in general not explicitly mention the associativity and commutativity isomorphisms. By the coherence theorem for AC tensor categories this will not cause any confusion. However, using inverses and commutativity isomorphisms together some- 
times introduces signs (automorphisms of order 2) and therefore in those cases it is important to keep track of all commutativities involved.

The abelian group of isomorphism classes of the Picard category $\mathcal{P}$ is denoted by $\pi_{0}(\mathcal{P})$ and the abelian group of automorphisms of $1_{\mathcal{P}}$ is denoted by $\pi_{1}(\mathcal{P})$. We note that for any object $L$ of $\mathcal{P}$ there is a canonical isomorphism $\operatorname{Aut}(L) \cong \operatorname{Aut}\left(1_{\mathcal{P}}\right)=$ $\pi_{1}(\mathcal{P})$ and that for two isomorphic objects $L$ and $M$ the set $\operatorname{Hom}(L, M)$ is a $\pi_{1}(\mathcal{P})$ torsor. Let $\psi$ be the commutativity constraint of $\mathcal{P}$. For any object $L$ of $\mathcal{P}$ we define $\varepsilon(L) \in \pi_{1}(\mathcal{P})$ as the automorphism $\psi_{L, L} \in \operatorname{Aut}(L \otimes L) \cong \pi_{1}(\mathcal{P})$. One can show that this induces a homomorphism $\varepsilon: \pi_{0}(\mathcal{P}) \rightarrow \pi_{1}(\mathcal{P})$ of order at most 2 .

Let $\mathcal{E}$ be an exact category and let $w$ be an $S Q$-class of morphisms in $\mathcal{E}$ (cf. [8, Definition 1.1]), for example the class 'iso' of all isomorphisms in $\mathcal{E}$. We let $\mathcal{E}_{w}$ denote the subcategory of $\mathcal{E}$ where morphisms are restricted to $w$. A determinant functor $\left(f_{1}, f_{2}\right)$ on $\mathcal{E}_{w}$ with values in the Picard category $\mathcal{P}$ consists of a functor $f_{1}: \mathcal{E}_{w} \rightarrow \mathcal{P}$ and an isomorphism $f_{2}(\Delta): f_{1}(Q) \rightarrow f_{1}(P) \otimes f_{1}(R)$ for each short exact sequence

$$
\Delta: 0 \longrightarrow P \longrightarrow Q \longrightarrow R \longrightarrow 0
$$

in $\mathcal{E}$. One requires $f_{2}$ to be natural with respect to morphisms of short exact sequences in $w^{3}$. Furthermore the data $\left(f_{1}, f_{2}\right)$ must satisfy three axioms (compatibility, associativity and commutativity) which are explained in [8, Definition 1.4]. To simplify the notation we will write $[\cdot]$ for both $f_{1}(\cdot)$ and $f_{2}(\cdot)$; the meaning will always be clear from the context. If 0 is a zero object in $\mathcal{E}$, then [0] has canonically the structure of a unit in $\mathcal{P}$ and therefore there exists a canonical isomorphism $[0] \rightarrow 1_{\mathcal{P}}$

Remark 2.1. One can show that the compatibility axiom in the definition of a determinant functor in $[\mathbf{8}]$ follows from the associativity axiom and is therefore redundant. Indeed, this follows easily by applying the associativity axiom to the diagram

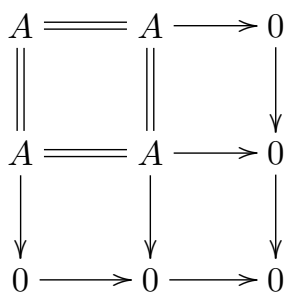

and using the fact that the unit structure on [0] is induced by the short exact sequence $0 \rightarrow 0 \rightarrow 0$. In this regard see also $[6, \S 4.6]$.

We write $\operatorname{det}\left(\mathcal{E}_{w}, \mathcal{P}\right)$ for the category of determinant functors on $\mathcal{E}_{w}$ with values in $\mathcal{P}$. If $\mathcal{P}$ and $\mathcal{Q}$ are two Picard categories then we write $\operatorname{Hom}^{\otimes}(\mathcal{P}, \mathcal{Q})$ for the category of monoidal functors (that is AC tensor functors in the terminology of $[\mathbf{8}]$ ). We recall that for any exact category $\mathcal{E}$ there exists a universal determinant functor $[\cdot]: \mathcal{E}_{\text {iso }} \rightarrow \mathcal{V}(\mathcal{E})$, that is a determinant functor such that for every Picard category $\mathcal{P}$ the induced functor $\operatorname{Hom}^{\otimes}(\mathcal{V}(\mathcal{E}), \mathcal{P}) \rightarrow \operatorname{det}\left(\mathcal{E}_{\text {iso }}, \mathcal{P}\right)$ is an equivalence of categories (see $[\mathbf{4}, \S 2.3]$ and $[\mathbf{6}, \S 4]$ for more details). The Picard category $\mathcal{V}(\mathcal{E})$ is called the category of virtual objects. There are canonical isomorphisms $\pi_{0}(\mathcal{V}(\mathcal{E})) \cong K_{0}(\mathcal{E})$ 
and $\pi_{1}(\mathcal{V}(\mathcal{E})) \cong K_{1}(\mathcal{E})$. If $\Lambda$ is a ring and $\mathcal{E}$ is equal to the category $\operatorname{PMod}(\Lambda)$ of finitely generated projective left $\Lambda$-modules, then we write $\mathcal{V}(\Lambda)$ for $\mathcal{V}(\mathcal{E})$. We remark that if $\Lambda$ is a commutative and noetherian ring then there is a natural determinant functor on $\operatorname{PMod}(\Lambda)_{\text {iso }}$ with values in the category of graded invertible $\Lambda$-modules (cf. $[\mathbf{9}])$. It is shown in $[\mathbf{4}, \S 2.5]$ that in many cases this determinant functor is universal and therefore provides an explicit construction of $\mathcal{V}(\Lambda)$.

A filtration $0=A_{0} \subseteq A_{1} \subseteq \cdots \subseteq A_{m}=P$ of an object $P$ in $\mathcal{E}$ is admissible if each inclusion $A_{i-1} \subseteq A_{i}$ is an admissible monomorphism. For such a filtration one has a canonical isomorphism $[P] \rightarrow \bigotimes_{i=1}^{m}\left[A_{i} / A_{i-1}\right]$. We will use [8, Proposition 1.9] to compare these isomorphisms for two compatible admissible filtrations of the same complex $P$. For the convenience of the reader we recall here the precise statement of this result.

Proposition 2.2. Let $0=A_{0} \subseteq A_{1} \subseteq \cdots \subseteq A_{m}=P$ and $0=B_{0} \subseteq B_{1} \subseteq \cdots \subseteq$ $B_{n}=P$ be compatible admissible filtrations of $P$ (see [8, Definition 1.8] for the definition of compatible). Then there is a commutative diagram

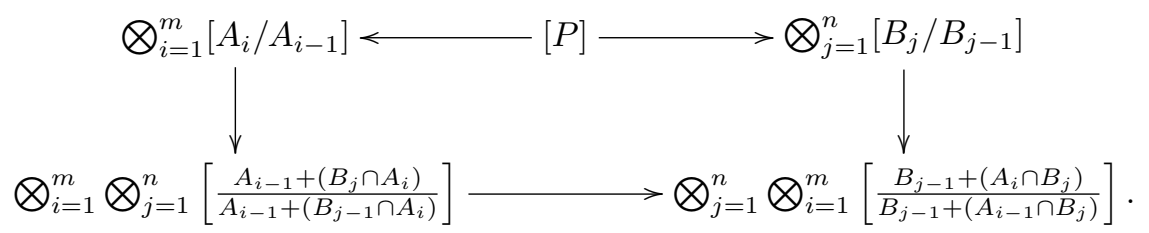

Here the vertical isomorphisms come from refining each quotient $A_{i} / A_{i-1}$ by the filtration

$$
A_{i-1}=A_{i-1}+\left(B_{0} \cap A_{i}\right) \subseteq A_{i-1}+\left(B_{1} \cap A_{i}\right) \subseteq \cdots \subseteq A_{i-1}+\left(B_{n} \cap A_{i}\right)=A_{i}
$$

and each quotient $B_{j} / B_{j-1}$ by the filtration

$$
B_{j-1}=B_{j-1}+\left(A_{0} \cap B_{j}\right) \subseteq B_{j-1}+\left(A_{1} \cap B_{j}\right) \subseteq \cdots \subseteq B_{j-1}+\left(A_{m} \cap B_{j}\right)=B_{j} .
$$

The bottom horizontal isomorphism is induced by the butterfly isomorphisms

$$
\frac{A_{i-1}+\left(B_{j} \cap A_{i}\right)}{A_{i-1}+\left(B_{j-1} \cap A_{i}\right)} \cong \frac{A_{i} \cap B_{j}}{\left(A_{i-1} \cap B_{j}\right)+\left(A_{i} \cap B_{j-1}\right)} \cong \frac{B_{j-1}+\left(A_{i} \cap B_{j}\right)}{B_{j-1}+\left(A_{i-1} \cap B_{j}\right)} .
$$

In this paper we are interested in determinant functors on certain categories of complexes. We write $\mathcal{C}^{b}(\mathcal{E})$ for the category of bounded cochain complexes of objects in $\mathcal{E}$ and 'qis' for the class of quasi-isomorphisms in $\mathcal{C}^{b}(\mathcal{E})$. Clearly $\mathcal{C}^{b}(\mathcal{E})$ is again an exact category and qis is an $S Q$-class of morphisms.

If $P$ is a complex then $P[1]$ denotes the shifted complex, that is $P[1]^{i}=P^{i+1}$ with differential $d_{P[1]}(p)=-d_{P}(p)$. For a map $a: P \rightarrow Q$ of complexes one defines the mapping cone cone $(a)$ to be the complex given by cone $(a)^{i}=Q^{i} \oplus P^{i+1}$ with differential $d_{\text {cone }(a)}(q, p)=\left(d_{Q}(q)+a(p),-d_{P}(p)\right)$. There is a short exact sequence of complexes $0 \rightarrow Q \rightarrow$ cone $(a) \rightarrow P[1] \rightarrow 0$ with maps given by the canonical injection and projection, that is $q \mapsto(q, 0)$ and $(q, p) \mapsto p$. If the complexes $P$ and $Q$ are in $\mathcal{C}^{b}(\mathcal{E})$, then so also is cone $(a)$.

For the rest of this section we let [.] be a determinant functor on $\mathcal{C}^{b}(\mathcal{E})_{\text {qis }}$ with values in $\mathcal{P}$. We remark that the main result of $[8]$ shows that giving such a de- 
terminant functor is essentially equivalent to giving a determinant functor on $\mathcal{E}_{\text {iso }}$ with values in $\mathcal{P}$, however we will not need this result until Section 5 . By definition of a Picard category, every object in $\mathcal{P}$ has an inverse. In general this inverse is not unique, but the next lemma shows that for every complex $P$ in $\mathcal{C}^{b}(\mathcal{E})$ there is a canonical inverse of $[P]$.

Lemma 2.3. For every complex $P$ in $\mathcal{C}^{b}(\mathcal{E})$ there is a canonical isomorphism

$$
\mu_{P}:[P] \otimes[P[1]] \longrightarrow 1_{\mathcal{P}} .
$$

If $a: P \stackrel{\sim}{\longrightarrow} Q$ is a quasi-isomorphism, then $\mu_{Q} \circ([a] \otimes[a[1]])=\mu_{P}$. The isomorphism $\mu$ is compatible with short exact sequences, and more generally if $0 \subseteq A_{0} \subseteq A_{1} \subseteq$ $\cdots \subseteq A_{m}=P$ is an admissible filtration then the diagram

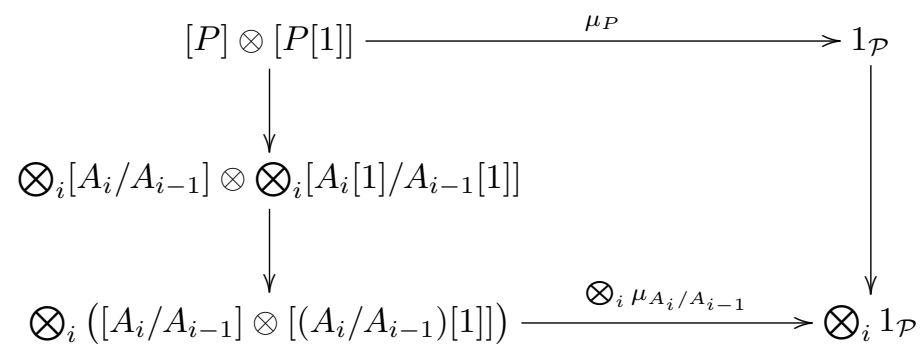

commutes; here $A_{i}[1] / A_{i-1}[1] \cong\left(A_{i} / A_{i-1}\right)[1]$ is the canonical isomorphism.

Proof. Let cone $\left(\operatorname{id}_{P}\right)$ be the mapping cone of the identity map $P \stackrel{\operatorname{id}_{P}}{\longrightarrow} P$. Then there is a canonical short exact sequence $0 \rightarrow P \rightarrow \operatorname{cone}\left(\operatorname{id}_{P}\right) \rightarrow P[1] \rightarrow 0$ and a quasi-isomorphism cone $\left(\operatorname{id}_{P}\right) \stackrel{\sim}{\longrightarrow} 0$. We define $\mu_{P}$ to be the composite isomorphism

$$
[P] \otimes[P[1]] \longrightarrow\left[\operatorname{cone}\left(\operatorname{id}_{P}\right)\right] \longrightarrow[0] \longrightarrow 1_{\mathcal{P}} .
$$

Note that a quasi-isomorphism $a: P \stackrel{\sim}{\longrightarrow} Q$ induces a quasi-isomorphism of mapping cones cone $\left(\operatorname{id}_{P}\right) \stackrel{\sim}{\longrightarrow} \operatorname{cone}\left(\operatorname{id}_{Q}\right)$ which immediately implies $\mu_{Q} \circ([a] \otimes[a[1]])=$ $\mu_{P}$. In addition, compatibility of this construction with short exact sequences follows from the fact that each short exact sequence of complexes $0 \rightarrow P \rightarrow Q \rightarrow R \rightarrow 0$ induces a short exact sequence of mapping cones $0 \rightarrow$ cone $\left(\operatorname{id}_{P}\right) \rightarrow$ cone $\left(\operatorname{id}_{Q}\right) \rightarrow$ cone $\left(\operatorname{id}_{R}\right) \rightarrow 0$. The proof of compatibility with admissible filtrations of arbitrary (finite) length is then reduced to the case of short exact sequences by an induction on the length of the filtration.

Let $P$ be a complex in $\mathcal{C}^{b}(\mathcal{E})$ and let - id $: P \rightarrow P$ denote the negative of the identity isomorphism. In $[\mathbf{6}, \S 4.9]$ it is shown that the automorphism $[-\mathrm{id}] \in$ Aut $([P]) \cong \pi_{1}(\mathcal{P})$ agrees with $\varepsilon([P]) \in \pi_{1}(\mathcal{P})$. Lemma 2.3 implies that $\varepsilon([P[i]])=$ $\varepsilon([P])$ for every complex $P$ and every integer $i$. To simplify the notation we will write $\varepsilon([P])$ for $\varepsilon([P[0]])$ if $P$ is an object in $\mathcal{E}$.

\section{Determinant functors and cohomology}

In this section we assume that $\mathcal{E}$ is an abelian category and that [.] is a determinant functor on $\mathcal{C}^{b}(\mathcal{E})_{\text {qis }}$ with values in a Picard category $\mathcal{P}$. For $P \in \mathcal{C}^{b}(\mathcal{E})$ we 
write $Z P, B P$ and $H P$ for the complexes of cycles, boundaries and cohomology of $P$ respectively, each with zero differentials. These complexes again belong to $\mathcal{C}^{b}(\mathcal{E})$.

Proposition 3.1. For every complex $P$ in $\mathcal{C}^{b}(\mathcal{E})$ there is a canonical isomorphism

$$
\eta_{P}:[P] \longrightarrow[H P] \text {. }
$$

If $a: P \stackrel{\sim}{\longrightarrow} Q$ is a quasi-isomorphism, then $\eta_{Q} \circ[a]=[H a] \circ \eta_{P}$.

Proof. We define $\eta_{P}$ as the composite isomorphism

$$
\begin{aligned}
{[P] } & \longrightarrow[B P] \otimes[H P] \otimes[B P[1]] \\
& \longrightarrow[H P]
\end{aligned}
$$

which is induced by the filtration

$$
0 \subseteq B P \subseteq Z P \subseteq P
$$

with quotients $B P / 0 \cong B P, Z P / B P \cong H P$ and $P / Z P \cong B P[1]$, and by the isomorphism $\mu_{B P}:[B P] \otimes[B P[1]] \rightarrow 1_{\mathcal{P}}$ from Lemma 2.3.

If $P=\left[P^{i} \stackrel{d^{i}}{\longrightarrow} P^{i+1}\right]$ is a complex of length at most 2 whose differential $d^{i}$ is a monomorphism then there is a canonical quasi-isomorphism $h_{P}: P \rightarrow H P$. From the commutative diagram of short exact sequences

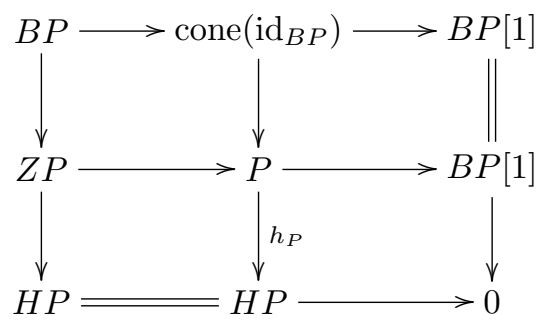

one then sees that $\eta_{P}=\left[h_{P}\right]:[P] \rightarrow[H P]$. This implies $\eta_{Q} \circ[a]=[H a] \circ \eta_{P}$ if $a: P \stackrel{\sim}{\longrightarrow} Q$ is a quasi-isomorphism and $P$ and $Q$ are both complexes of length at most 2 with injective differential. The general case follows by induction on the length of $P$ using the (good) truncation filtration as in the proof of $[\mathbf{8}$, Proposition $3.2]$.

Remark 3.2. Let $i_{P}: B P \rightarrow Z P$ denote the canonical inclusion. In $[\mathbf{8}, \S 3]$, Knudsen considers the composite isomorphism

$$
[P] \longrightarrow[Z P] \otimes[B P[1]] \longrightarrow\left[\operatorname{cone}\left(i_{P}\right)\right] \longrightarrow[H P]
$$

which is induced by the short exact sequences $0 \rightarrow Z P \rightarrow P \rightarrow B P[1] \rightarrow 0$ and $0 \rightarrow Z P \rightarrow \operatorname{cone}\left(i_{P}\right) \rightarrow B P[1] \rightarrow 0$ and by the canonical quasi-isomorphism cone $\left(i_{P}\right) \stackrel{\sim}{\longrightarrow} H P$. It is not difficult to show that this isomorphism agrees with $\eta_{P}$ in Proposition 3.1. Therefore the naturality of $\eta$ with respect to quasi-isomorphisms also follows from [8, Proposition 3.3].

For a short exact sequence

$$
\Delta: 0 \longrightarrow P \stackrel{a}{\longrightarrow} Q \stackrel{b}{\longrightarrow} R \longrightarrow 0
$$


in $\mathcal{C}^{b}(\mathcal{E})$ one has an isomorphism $[\Delta]:[Q] \rightarrow[P] \otimes[R]$. Using the identifications from Proposition 3.1 we obtain an induced isomorphism $[H Q] \rightarrow[H P] \otimes[H R]$. We shall now give a description of this isomorphism in purely cohomological terms.

Let $\partial: H R \rightarrow H P[1]$ be the connecting homomorphism in the long exact cohomology sequence induced by $\Delta$. Then the long exact cohomology sequence can be written as the exact sequence of complexes

$$
0 \longrightarrow \operatorname{ker}(H a) \longrightarrow H P \stackrel{H a}{\longrightarrow} H Q \stackrel{H b}{\longrightarrow} H R \stackrel{\partial}{\longrightarrow} \operatorname{ker}(H a)[1] \longrightarrow 0 .
$$

We define $[H \Delta]:[H Q] \rightarrow[H P] \otimes[H R]$ to be the isomorphism

$$
\begin{aligned}
{[H Q] } & \longrightarrow[H Q] \otimes[\operatorname{ker}(H a)] \otimes[\operatorname{ker}(H a)[1]] \\
& \longrightarrow[H P] \otimes[H R]
\end{aligned}
$$

which is induced by the isomorphism $\mu_{\operatorname{ker}(H a)}^{-1}: 1_{\mathcal{P}} \rightarrow[\operatorname{ker}(H a)] \otimes[\operatorname{ker}(H a)[1]]$ and by the exact sequence (1).

Theorem 3.3. The isomorphism $[H \Delta]:[H Q] \rightarrow[H P] \otimes[H R]$ is the unique map making the diagram

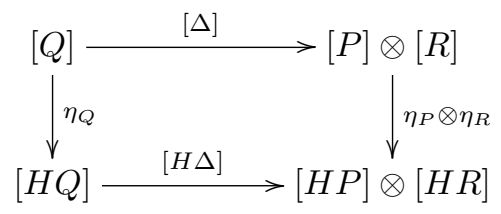

commutative.

Proof. The idea of this proof is to introduce two filtrations on the complex $Q$, apply Proposition 2.2 to these filtrations and then identify certain of the resulting subquotients with the objects in the exact sequence (1).

The first filtration is

$$
0 \subseteq B Q \subseteq Z Q \subseteq Q
$$

coming from the boundaries and cycles of Q. The second filtration is

$$
0 \subseteq B P \subseteq Z P \subseteq P \subseteq b^{-1}(B R) \subseteq b^{-1}(Z R) \subseteq Q
$$

coming from the boundaries and cycles of $P$ and from the preimage of the filtration $0 \subseteq B R \subseteq Z R \subseteq R$ under the map $b: Q \rightarrow R$. Obviously there are isomorphisms $b^{-1}(B R) / P \cong B R, b^{-1}(Z R) / b^{-1}(B R) \cong H R$ and $Q / b^{-1}(Z R) \cong R / Z R$.

By refining the filtration (2) as in Proposition 2.2 we obtain

$$
\begin{gathered}
0 \subseteq B P \subseteq Z P \cap B Q \subseteq B Q, \\
B Q \subseteq Z P+B Q \subseteq Z Q, \\
Z Q \subseteq P+Z Q \subseteq b^{-1}(Z R) \subseteq Q
\end{gathered}
$$


which gives (using the identifications in the table below)

$$
\begin{aligned}
{[Q] \cong } & {[B Q] \otimes[H Q] \otimes[Q / Z Q] } \\
\cong & ([B P] \otimes[\operatorname{ker}(H a)] \otimes[B R]) \\
& \otimes([\operatorname{ker}(H b)] \otimes[\operatorname{im}(H b)]) \\
& \otimes([P / Z P] \otimes[H R / \operatorname{im}(H b)] \otimes[R / Z R]) .
\end{aligned}
$$

By refining the filtration (3) as in Proposition 2.2 we obtain

$$
\begin{gathered}
0 \subseteq B P, \quad B P \subseteq Z P \cap B Q \subseteq Z P, \quad Z P \subseteq P, \\
P \subseteq b^{-1}(B R), \quad b^{-1}(B R) \subseteq P+Z Q \subseteq b^{-1}(Z R), \quad b^{-1}(Z R) \subseteq Q
\end{gathered}
$$

which gives (again using the table below)

$$
\begin{aligned}
{[Q] \cong } & {[B P] \otimes[H P] \otimes[P / Z P] } \\
& \otimes[B R] \otimes[H R] \otimes[R / Z R] \\
\cong & ([B P]) \otimes([\operatorname{ker}(H a)] \otimes[\operatorname{ker}(H b)]) \otimes([P / Z P]) \\
& \otimes([B R]) \otimes([\operatorname{im}(H b)] \otimes[H R / \operatorname{im}(H b)]) \otimes([R / Z R]) .
\end{aligned}
$$

By Proposition 2.2 the isomorphisms (4) and (5) agree after reordering the terms on the right hand sides.

In the following table we summarise the identifications used. The three objects in each row are isomorphic. The obvious isomorphism between the subquotient in the first column and the subquotient in the second column is the butterfly isomorphism of Proposition 2.2, and each of these subquotients is canonically isomorphic to the object in the third column.

\begin{tabular}{c|c|c} 
subquotient in (4) & subquotient in (5) & identified with \\
\hline$B P / 0$ & $B P / 0$ & $B P$ \\
$(Z P \cap B Q) / B P$ & $(Z P \cap B Q) / B P$ & $\operatorname{ker}(H a)$ \\
$B Q /(Z P \cap B Q)$ & $b^{-1}(B R) / P$ & $B R$ \\
\hline$(Z P+B Q) / B Q$ & $Z P /(Z P \cap B Q)$ & $\operatorname{ker}(H b)$ \\
$Z Q /(Z P+B Q)$ & $(P+Z Q) / b^{-1}(B R)$ & $\operatorname{im}(H b)$ \\
\hline$(P+Z Q) / Z Q$ & $P / Z P$ & $P / Z P$ \\
$b^{-1}(Z R) /(P+Z Q)$ & $b^{-1}(Z R) /(P+Z Q)$ & $H R / \operatorname{im}(H b)$ \\
$Q / b^{-1}(Z R)$ & $Q / b^{-1}(Z R)$ & $R / Z R$
\end{tabular}

The differential $d: Q \rightarrow Q[1]$ induces an isomorphism $Q / Z Q \stackrel{\cong}{\longrightarrow} B Q[1]$. We therefore obtain an isomorphism $[B Q] \otimes[Q / Z Q] \rightarrow[B Q] \otimes[B Q[1]] \rightarrow 1_{\mathcal{P}}$ where the second map is $\mu_{B Q}$. More generally, $d$ induces an isomorphism from the filtration $\frac{Z Q}{Z Q} \subseteq \frac{P+Z Q}{Z Q} \subseteq \frac{b^{-1}(Z R)}{Z Q} \subseteq \frac{Q}{Z Q}$ to the filtration $0[1] \subseteq B P[1] \subseteq(Z P \cap B Q)[1] \subseteq$ $B Q[1]$, and we obtain isomorphisms $\left[\frac{B P}{0}\right] \otimes\left[\frac{P+Z Q}{Z Q}\right] \rightarrow\left[\frac{B P}{0}\right] \otimes\left[\frac{B P}{0}[1]\right] \rightarrow 1_{\mathcal{P}}$, etc. In Lemma 2.3 we showed that $\mu$ is compatible with filtrations which gives the commutativity of the upper square in diagram (7) below.

We obtain the lower commutative square in diagram (7) by identifying each subquotient with the corresponding object in the third column of the table above. 
Thus the bottom horizontal map is given by $[B P] \otimes\left[\frac{P}{Z P}\right] \rightarrow[B P] \otimes[B P[1]] \rightarrow 1_{\mathcal{P}}$, etc., where the isomorphisms

$$
P / Z P \stackrel{\cong}{\longrightarrow} B P[1], \quad H R / \operatorname{im}(H b) \stackrel{\cong}{\longrightarrow} \operatorname{ker}(H a)[1], \quad R / Z R \stackrel{\cong}{\longrightarrow} B R[1]
$$

are induced from the differential $d$ on the subquotients of $Q$. One easily checks that the first and third isomorphism in (6) agree with the isomorphism induced by the differential of $P$ and $R$ respectively, and that the second isomorphism is the connecting homomorphism $\partial$ of the long exact cohomology sequence.

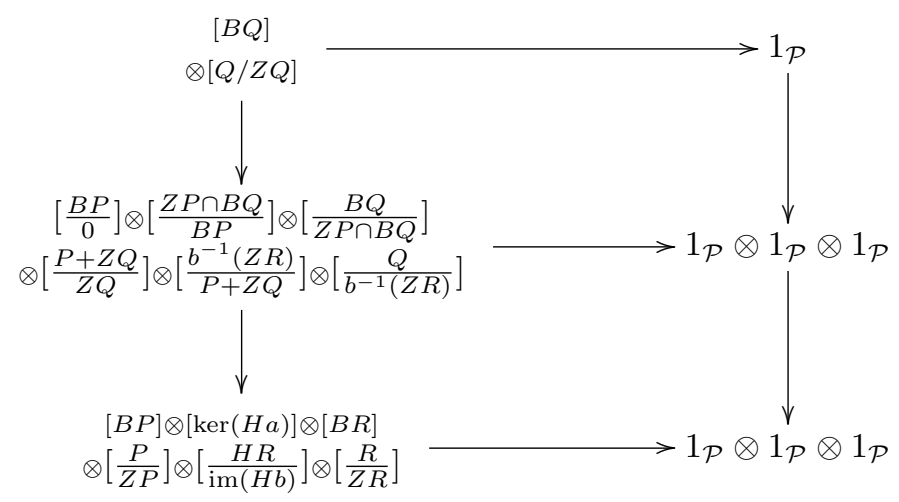

Now consider the following diagram.

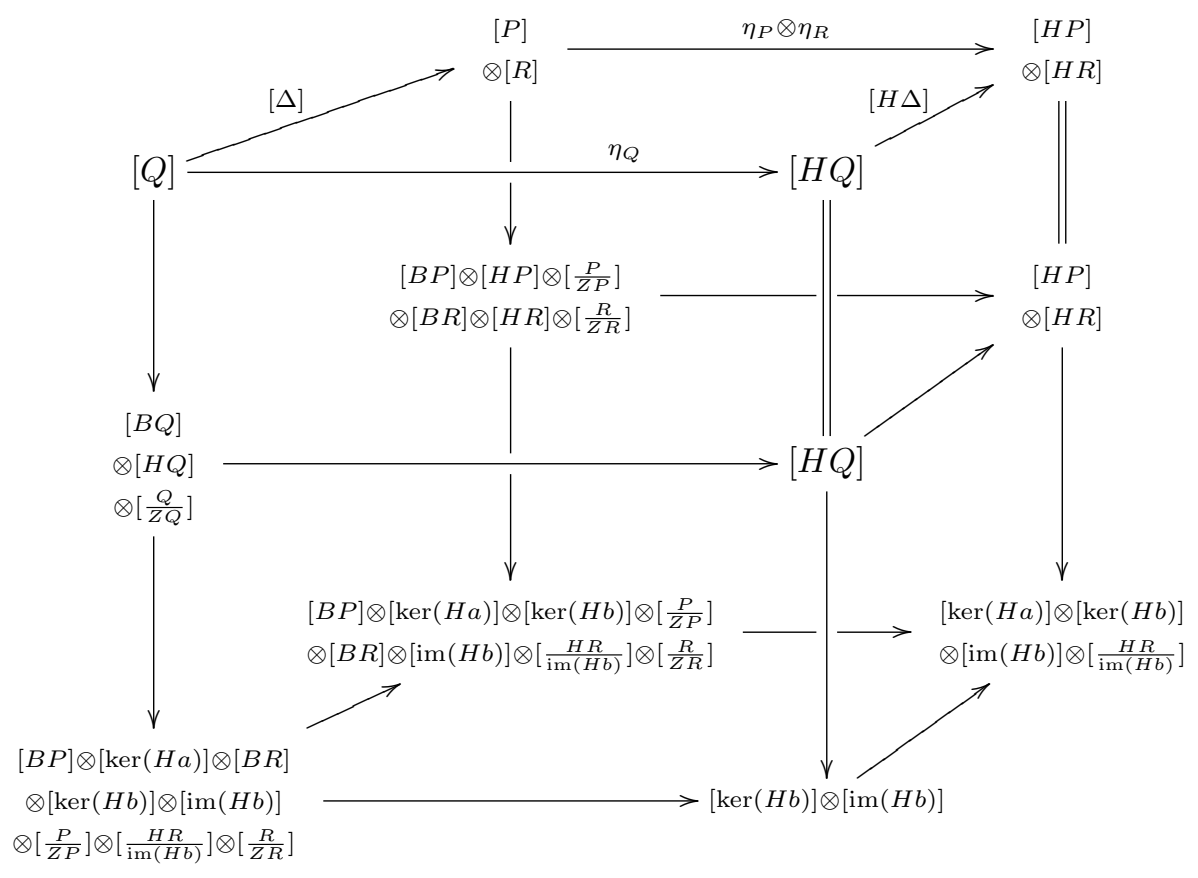

The left face of this diagram is commutative because the isomorphisms (4) and 
(5) agree. The right face is commutative by the definition of $[H \Delta]$. The upper front face and upper back face are commutative by the definition of the maps $\eta_{P}:[P] \rightarrow[H P], \eta_{R}:[R] \rightarrow[H R]$ and $\eta_{Q}:[Q] \rightarrow[H Q]$. The commutativity of the lower front face follows from (7). The lower back face and the bottom face are obviously commutative. The resulting commutativity of the top face is precisely the statement of the theorem.

As an immediate consequence of Theorem 3.3 we deduce the following result.

Corollary 3.4. The functor $[\cdot] \circ H: \mathcal{C}^{b}(\mathcal{E})_{\text {qis }} \rightarrow \mathcal{P}$ together with the isomorphism $[H \Delta]$ for each short exact sequence $\Delta$ in $\mathcal{C}^{b}(\mathcal{E})$ form a determinant functor, and $\eta:[\cdot] \rightarrow[\cdot] \circ H$ is an isomorphism of determinant functors.

We now end this section by proving a result which will be useful in $\S 5$.

Lemma 3.5. Let $P$ be a complex in $\mathcal{C}^{b}(\mathcal{E})$ and identify $H(P[1])$ and $(H P)[1]$ (in the obvious way). Then the diagram



is commutative.

Proof. Consider the diagram

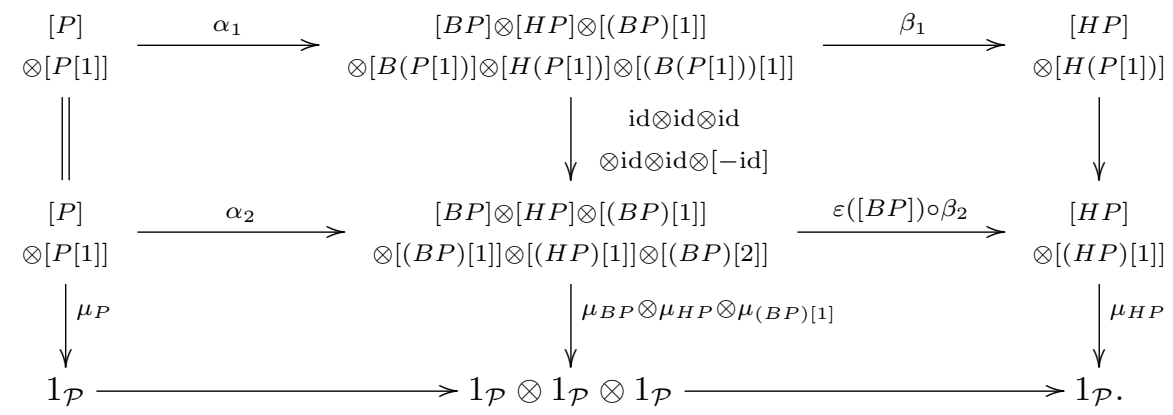

The map $\alpha_{1}$ is induced by the filtration $0 \subseteq B P \subseteq Z P \subseteq P$ with quotients $B P, H P$ and $(B P)[1]$, and by the filtration $0 \subseteq B(P[1]) \subseteq Z(P[1]) \subseteq P[1]$ with quotients $B(P[1]), H(P[1])$ and $(B(P[1]))[1]$.

For the map $\alpha_{2}$ we use the same filtration of $P$ as above and the filtration $0 \subseteq(B P)[1] \subseteq(Z P)[1] \subseteq P[1]$ on $P[1]$ which is obtained by shifting the filtration on $P$. The quotients of this filtration on $P[1]$ are identified with $(B P)[1],(H P)[1]$ and $(B P)[2]$ by shifting the identifications for the quotients of $P$. There are canonical isomorphisms $B(P[1]) \cong(B P)[1]$ and $(B(P[1]))[1] \cong(B P)[2]$ but to ensure commutativity of the upper left hand square we need the determinant of the negative of this isomorphism, i.e. $[-\mathrm{id}]:[(B(P[1]))[1]] \rightarrow[(B P)[2]]$. This is because the isomorphism $P[1] / Z(P[1]) \rightarrow(B(P[1]))[1]$ used for the map $\alpha_{1}$ is induced by the 
differential $d_{P[1]}$, whereas the isomorphism $P[1] /(Z P)[1] \rightarrow(B P)[2]$ used for the map $\alpha_{2}$ is induced by the differential $d_{P}$.

The compatibility of the isomorphism $\mu$ with filtrations implies the commutativity of the lower left hand square of the diagram. Note that in the vertical arrow marked $\mu_{B P} \otimes \mu_{H P} \otimes \mu_{(B P)[1]}$ the map $\mu_{B P}:[B P] \otimes[(B P)[1]] \rightarrow 1_{\mathcal{P}}$ refers to $[(B P)[1]]$ in the second row and $\mu_{(B P)[1]}:[(B P)[1]] \otimes[(B P)[2]] \rightarrow 1_{\mathcal{P}}$ refers to $[(B P)[1]]$ in the first row.

The map $\beta_{1}$ is obtained by applying $\mu_{B P}:[B P] \otimes[(B P)[1]] \rightarrow 1_{\mathcal{P}}$ and $\mu_{B(P[1])}$ : $[B(P[1])] \otimes[(B(P[1]))[1]] \rightarrow 1_{\mathcal{P}}$. Similarly the map $\beta_{2}$ is obtained by applying $\mu_{B P}:[B P] \otimes[(B P)[1]] \rightarrow 1_{\mathcal{P}}$ (referring to $[(B P)[1]]$ in the first row) and $\mu_{(B P)[1]}$ : $[(B P)[1]] \otimes[(B P)[2]] \rightarrow 1_{\mathcal{P}}$ (referring to $[(B P)[1]]$ in the second row). Commutativity of the upper right hand square is immediate because -id acting on $(B P)[2]$ gives the automorphism $\varepsilon([B P])$. Finally, the commutativity of the lower right hand square follows directly from the definition of $\varepsilon([B P])$.

Thus the above diagram is commutative. Since $\beta_{1} \circ \alpha_{1}=\eta_{P} \otimes \eta_{P[1]}$ this shows the commutativity of (8).

\section{Decomposition into even and odd degree parts}

In this section we prove various technical results relating the determinant of a complex to the determinants of its even and odd degree parts. This will be needed for our applications to Euler characteristics in $\S 5$ and $\S 6$. We let $\mathcal{E}$ be an exact category and [.] a determinant functor on $\mathcal{C}^{b}(\mathcal{E})_{\text {qis }}$ with values in a Picard category $\mathcal{P}$.

Lemma 4.1. For every complex $P$ in $\mathcal{C}^{b}(\mathcal{E})$ there is a canonical isomorphism

$$
\sigma_{P}:[P] \longrightarrow[P[2]] \text {. }
$$

If $a: P \stackrel{\sim}{\longrightarrow} Q$ is a quasi-isomorphism then $\sigma_{Q} \circ[a]=[a[2]] \circ \sigma_{P}$. The isomorphism $\sigma$ is compatible with short exact sequences, and more generally if $0=A_{0} \subseteq A_{1} \subseteq$ $\cdots \subseteq A_{m}=P$ is an admissible filtration, then the diagram



commutes, where for the bottom map we have used the canonical isomorphism $\left(A_{i} / A_{i-1}\right)[2] \cong A_{i}[2] / A_{i-1}[2]$.

Proof. We define $\sigma_{P}$ as the composite isomorphism

$$
\begin{aligned}
{[P] } & \longrightarrow[P] \otimes([P[1]] \otimes[P[2]]) \\
& \longrightarrow([P] \otimes[P[1]]) \otimes[P[2]] \\
& \longrightarrow[P[2]]
\end{aligned}
$$

where we have first applied $\mu_{P[1]}^{-1}: 1_{\mathcal{P}} \rightarrow[P[1]] \otimes[P[2]]$ and then $\mu_{P}:[P] \otimes$ 
$[P[1]] \rightarrow 1_{\mathcal{P}}$. Compatibility with quasi-isomorphisms and filtrations follows from the corresponding properties of $\mu_{P[1]}$ and $\mu_{P}$ (cf. Lemma 2.3).

Lemma 4.2. For every complex $P$ in $\mathcal{C}^{b}(\mathcal{E})$ the diagram



is commutative, where $\psi$ denotes the commutativity constraint.

Proof. We will show the following more general result. Let $L_{0}, L_{1}, L_{2}$ be objects in $\mathcal{P}$ and $\mu_{i}: L_{i} \otimes L_{i+1} \rightarrow 1_{\mathcal{P}}$ isomorphisms for $i=0,1$. If $\sigma_{0}: L_{0} \rightarrow L_{2}$ is defined by $L_{0} \rightarrow L_{0} \otimes\left(L_{1} \otimes L_{2}\right) \rightarrow\left(L_{0} \otimes L_{1}\right) \otimes L_{2} \rightarrow L_{2}$, then

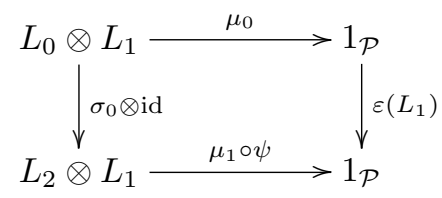

commutes. Obviously this will imply the statement in the lemma.

An argument similar to [8, Proposition A.14] shows that the map

$L_{1} \longrightarrow 1_{\mathcal{P}} \otimes L_{1} \stackrel{\mu_{1}^{-1} \otimes \mathrm{id}}{\longrightarrow} L_{1} \otimes L_{2} \otimes L_{1} \stackrel{\mathrm{id} \otimes \psi}{\longrightarrow} L_{1} \otimes L_{1} \otimes L_{2} \stackrel{\mathrm{id} \otimes \mu_{1}}{\longrightarrow} L_{1} \otimes 1_{\mathcal{P}} \longrightarrow L_{1}$

is $\varepsilon\left(L_{1}\right)$. Tensoring with $L_{0}$ on the left gives the commutative triangle in the following diagram (where we omit isomorphisms of the form $L_{0} \otimes 1_{\mathcal{P}} \cong L_{0}$ etc.).

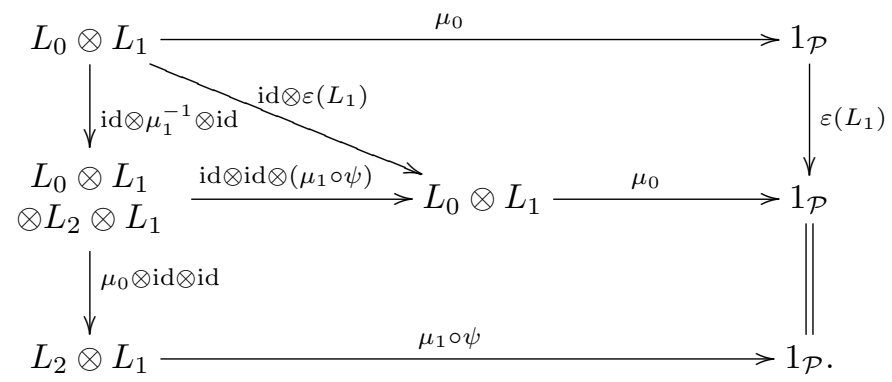

By definition of the isomorphism $\operatorname{Aut}\left(L_{1}\right) \cong \operatorname{Aut}\left(1_{\mathcal{P}}\right)$, the upper trapezium is commutative. The commutativity of the bottom rectangle is obvious.

Therefore diagram (9) is commutative as claimed.

Corollary 4.3. For every complex $P$ in $\mathcal{C}^{b}(\mathcal{E})$ the following diagram is commutative




Proof. This follows from Lemma 4.2 applied to the complexes $P$ and $P[1]$ and the observation that $\varepsilon([P[1]]) \circ \varepsilon([P])=\mathrm{id}_{1_{\mathcal{P}}}$.

For a complex $P$ in $\mathcal{C}^{b}(\mathcal{E})$ we define the objects $P^{\mathrm{ev}}$ and $P^{\mathrm{od}}$ in $\mathcal{E}$ by $P^{\mathrm{ev}}=$ $\bigoplus_{i \text { even }} P^{i}$ and $P^{\text {od }}=\bigoplus_{i \text { odd }} P^{i}$. Obviously these operations give exact functors $\mathcal{C}^{b}(\mathcal{E}) \rightarrow \mathcal{E}$.

Proposition 4.4. For every complex $P$ in $\mathcal{C}^{b}(\mathcal{E})$ there is a canonical isomorphism

$$
\pi_{P}:[P] \longrightarrow\left[P^{\mathrm{ev}}[0]\right] \otimes\left[P^{\mathrm{od}}[1]\right] .
$$

If $a: P \stackrel{\cong}{\longrightarrow} Q$ is an isomorphism, then $\pi_{Q} \circ[a]=\left(\left[a^{\mathrm{ev}}[0]\right] \otimes\left[a^{\mathrm{od}}[1]\right]\right) \circ \pi_{P}$. The isomorphism $\pi$ is compatible with short exact sequences, and more generally if $0=$ $A_{0} \subseteq A_{1} \subseteq \cdots \subseteq A_{m}=P$ is an admissible filtration, then the following diagram commutes



where for the right vertical maps we have used the induced admissible filtrations $0=A_{0}^{\mathrm{ev}} \subseteq A_{1}^{\mathrm{ev}} \subseteq \cdots \subseteq A_{m}^{\mathrm{ev}}=P^{\mathrm{ev}}$ and $0=A_{0}^{\text {od }} \subseteq A_{1}^{\text {od }} \subseteq \cdots \subseteq A_{m}^{\text {od }}=P^{\text {od }}$ and the canonical isomorphisms $A_{i}^{\text {ev }} / A_{i-1}^{\text {ev }} \cong\left(A_{i} / A_{i-1}\right)^{\text {ev }}$ and $A_{i}^{\text {od }} / A_{i-1}^{\text {od }} \cong\left(A_{i} / A_{i-1}\right)^{\text {od }}$.

Proof. We consider the brutal truncation filtration (cf. [8, Definition 2.17])

$$
\cdots \subseteq \sigma^{\geqslant i+1} P \subseteq \sigma^{\geqslant i} P \subseteq \sigma^{\geqslant i-1} P \subseteq \cdots \subseteq P .
$$

This filtration is admissible and has quotients $\sigma^{\geqslant i} P / \sigma^{\geqslant i+1} P \cong P^{i}[-i]$. We define $\pi_{P}$ as the composite isomorphism

$$
\begin{aligned}
{[P] } & \longrightarrow \bigotimes_{i}\left[P^{i}[-i]\right] \\
& \longrightarrow \bigotimes_{i \text { even }}\left[P^{i}[0]\right] \otimes \bigotimes_{i \text { odd }}\left[P^{i}[1]\right] \\
& \longrightarrow\left[P^{\mathrm{ev}}[0]\right] \otimes\left[P^{\text {od }}[1]\right]
\end{aligned}
$$

where for the second map we repeatedly applied the isomorphism $\sigma$ from Lemma 4.1 .

The compatibility with isomorphisms of complexes is obvious. Since the filtration $0=A_{0} \subseteq A_{1} \subseteq \cdots \subseteq A_{m}=P$ and the brutal truncation filtration of $P$ are compatible, we can apply Proposition 2.2. Together with the statement about filtrations in Lemma 4.1 this easily implies the compatibility of $\pi$ with respect to filtrations. 
Lemma 4.5. For every complex $P$ the diagram

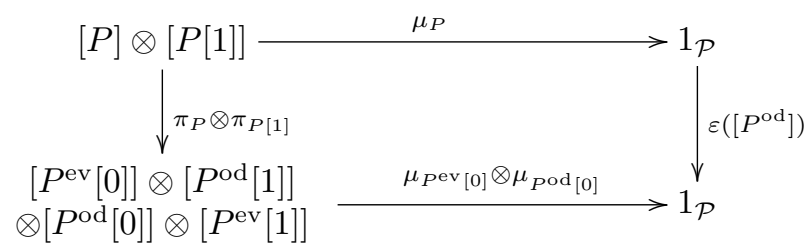

is commutative where for the left map we have used $P[1]^{\mathrm{ev}}=P^{\mathrm{od}}$ and $P[1]^{\mathrm{od}}=P^{\mathrm{ev}}$ and for the bottom map we have used $\mu_{P^{\mathrm{ev}}[0]} \otimes \mu_{P^{\mathrm{od}}[0]}$ after reordering the terms.

Proof. Specialising the diagram in Lemma 2.3 to the brutal truncation filtration gives the commutative diagram

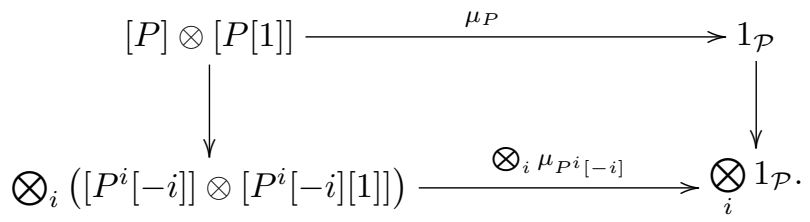

The top square in diagram (11) below is commutative by Corollary 4.3 and the compatibility of the isomorphism $\mu$ with respect to direct sums. Commutativity of the bottom square follows from Lemma 4.2.

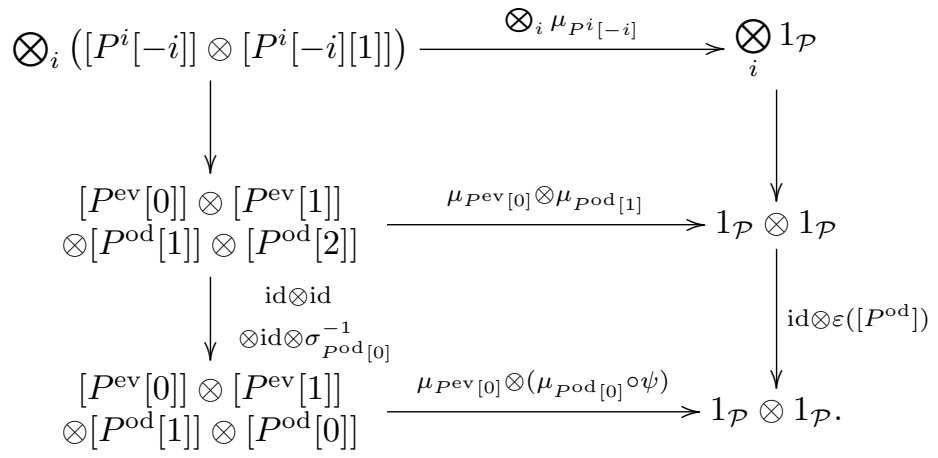

Diagrams (10) and (11) show the lemma.

\section{Euler characteristics}

In the sequel we fix rings $\Lambda$ and $\Sigma$ and a ring homomorphism $\Lambda \rightarrow \Sigma$ such that $\Sigma$ is flat as a right $\Lambda$-module. We assume that $\Sigma$ is noetherian and regular, that is every finitely generated $\Sigma$-module has finite projective dimension. We write $P_{\Sigma}=\Sigma \otimes_{\Lambda} P$ for the scalar extension if $P$ is a $\Lambda$-module or a complex of $\Lambda$-modules. We recall that there is a canonical exact sequence of algebraic $K$-groups

$$
K_{1}(\Lambda) \longrightarrow K_{1}(\Sigma) \stackrel{\delta_{\Lambda, \Sigma}^{1}}{\longrightarrow} K_{0}(\Lambda, \Sigma) \stackrel{\delta_{\Lambda, \Sigma}^{0}}{\longrightarrow} K_{0}(\Lambda) \longrightarrow K_{0}(\Sigma),
$$


where $K_{0}(\Lambda, \Sigma)$ is the relative algebraic $K$-group as defined by Swan in [12, p. 215]. We recall also that elements in $K_{0}(\Lambda, \Sigma)$ are represented by triples $(P, g, Q)$ where $P$ and $Q$ belong to the category $\operatorname{PMod}(\Lambda)$ of finitely generated projective $\Lambda$-modules and $g: P_{\Sigma} \rightarrow Q_{\Sigma}$ is an isomorphism of $\Sigma$-modules.

The scalar extension functor $\operatorname{PMod}(\Lambda) \rightarrow \operatorname{PMod}(\Sigma), P \mapsto P_{\Sigma}$, is exact and by the universal property of virtual objects it therefore induces a monoidal functor $\mathcal{V}(\Lambda) \rightarrow \mathcal{V}(\Sigma)$ (unique up to natural isomorphism). We fix such a monoidal functor which again we will write as $L \mapsto L_{\Sigma}$. Furthermore we fix an isomorphism in $\operatorname{det}\left(\operatorname{PMod}(\Lambda)_{\text {iso }}, \mathcal{V}(\Sigma)\right)$ from the determinant functor $\operatorname{PMod}(\Lambda)_{\text {iso }} \rightarrow \mathcal{V}(\Lambda) \rightarrow \mathcal{V}(\Sigma)$ to the determinant functor $\operatorname{PMod}(\Lambda)_{\text {iso }} \rightarrow \operatorname{PMod}(\Sigma)_{\text {iso }} \rightarrow \mathcal{V}(\Sigma)$ (the existence of such an isomorphism also follows from the universal property).

We fix a unit $1_{\mathcal{V}(\Sigma)}$ in $\mathcal{V}(\Sigma)$ and define a tensor category $\mathcal{V}(\Lambda, \Sigma)$ as follows. Objects in $\mathcal{V}(\Lambda, \Sigma)$ are pairs $(L, \lambda)$ with $L$ an object in $\mathcal{V}(\Lambda)$ and $\lambda$ an isomorphism $L_{\Sigma} \rightarrow 1_{\mathcal{V}(\Sigma)}$ in $\mathcal{V}(\Sigma)$. A morphism from $(L, \lambda)$ to $(M, \mu)$ is an isomorphism $\alpha: L \rightarrow$ $M$ in $\mathcal{V}(\Lambda)$ such that $\mu \circ \alpha_{\Sigma}=\lambda$ in $\mathcal{V}(\Sigma)$. The product of $(L, \lambda)$ and $(M, \mu)$ is $(L \otimes M, \nu)$ where $\nu:(L \otimes M)_{\Sigma} \rightarrow 1_{\mathcal{V}(\Sigma)}$ is the isomorphism

$$
(L \otimes M)_{\Sigma} \longrightarrow L_{\Sigma} \otimes M_{\Sigma} \stackrel{\lambda \otimes \mu}{\longrightarrow} 1_{\mathcal{V}(\Sigma)} \otimes 1_{\mathcal{V}(\Sigma)} \longrightarrow 1_{\mathcal{V}(\Sigma)}
$$

Lemma 5.1. The tensor category $\mathcal{V}(\Lambda, \Sigma)$ is a Picard category (with respect to natural associativity and commutativity constraints) and there is an isomorphism $\pi_{0} \mathcal{V}(\Lambda, \Sigma) \cong K_{0}(\Lambda, \Sigma)$.

Proof. The proof is similar to the proof of [4, Proposition 2.5]. Let $\mathcal{P}_{0}$ be the Picard category with unique object $1_{\mathcal{P}_{0}}$ and trivial automorphism group $\operatorname{Aut}_{\mathcal{P}_{0}}\left(1_{\mathcal{P}_{0}}\right)$. Then $\mathcal{V}(\Lambda, \Sigma)$ is the fibre product

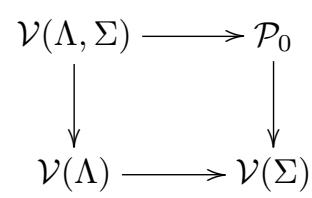

where $\mathcal{P}_{0} \rightarrow \mathcal{V}(\Sigma)$ maps $1_{\mathcal{P}_{0}}$ to $1_{\mathcal{V}(\Sigma)}$. This implies that $\mathcal{V}(\Lambda, \Sigma)$ is a Picard category with respect to the associativity and commutativity constraints that are induced by those of $\mathcal{V}(\Lambda)$ and $\mathcal{V}(\Sigma)$.

We define a homomorphism $\xi: K_{0}(\Lambda, \Sigma) \rightarrow \pi_{0} \mathcal{V}(\Lambda, \Sigma)$ by sending a generator $(P, g, Q)$ to the isomorphism class of $\left([P] \otimes[Q]^{-1}, \lambda\right)$ where $\lambda:\left([P] \otimes[Q]^{-1}\right)_{\Sigma} \rightarrow$ $1_{\mathcal{V}(\Sigma)}$ is the isomorphism

$$
\left([P] \otimes[Q]^{-1}\right)_{\Sigma} \longrightarrow\left[P_{\Sigma}\right] \otimes\left[Q_{\Sigma}\right]^{-1} \stackrel{[g] \otimes \mathrm{id}}{\longrightarrow}\left[Q_{\Sigma}\right] \otimes\left[Q_{\Sigma}\right]^{-1} \longrightarrow 1_{\mathcal{V}(\Sigma)}
$$

(Given the defining relations of $K_{0}(\Lambda, \Sigma)$ [12, p. 215], it is a straightforward exercise to show that $\xi$ is well-defined.) Now the exact sequence (12) and the Mayer-Vietoris 
sequence of the fibre product fit into a commutative diagram

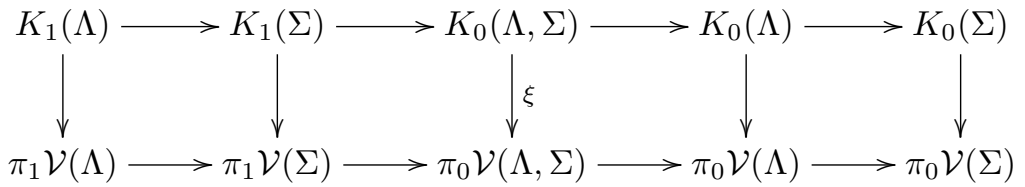

in which all unlabelled vertical arrows represent the canonical isomorphisms. From the 5-Lemma we may therefore deduce that $\xi$ is itself an isomorphism.

We note that if $\alpha \in K_{1}(\Sigma) \cong \pi_{1} \mathcal{V}(\Sigma)$, then $\delta_{\Lambda, \Sigma}^{1}(\alpha) \in K_{0}(\Lambda, \Sigma)$ is represented by the isomorphism class of $\left(1_{\mathcal{V}(\Lambda)}, \lambda\right) \in \mathcal{V}(\Lambda, \Sigma)$ where $1_{\mathcal{V}(\Lambda)}$ is a unit in $\mathcal{V}(\Lambda)$ and $\lambda$ is the isomorphism $\left(1_{\mathcal{V}(\Lambda)}\right)_{\Sigma} \rightarrow 1_{\mathcal{V}(\Sigma)} \stackrel{\alpha}{\rightarrow} 1_{\mathcal{V}(\Sigma)}$.

Next we consider the extension of determinant functors to certain categories of complexes. By [8, Theorem 2.3], the restriction functor

$$
\operatorname{det}\left(\mathcal{C}^{b}(\operatorname{PMod}(\Lambda))_{\text {qis }}, \mathcal{P}\right) \longrightarrow \operatorname{det}\left(\operatorname{PMod}(\Lambda)_{\text {iso }}, \mathcal{P}\right)
$$

is an equivalence of categories. The following result shows that for the regular ring $\Sigma$ one can even consider bounded complexes of objects in the category $\operatorname{Mod}(\Sigma)$ of finitely generated $\Sigma$-modules.

Lemma 5.2. For any Picard category $\mathcal{P}$ the restriction functor

$$
\operatorname{det}\left(\mathcal{C}^{b}(\operatorname{Mod}(\Sigma))_{\text {qis }}, \mathcal{P}\right) \longrightarrow \operatorname{det}\left(\operatorname{PMod}(\Sigma)_{\text {iso }}, \mathcal{P}\right)
$$

is an equivalence of categories.

Proof. We recall that by $\left[8\right.$, Theorem 2.3], the functor $\operatorname{det}\left(\mathcal{C}^{b}(\operatorname{Mod}(\Sigma))_{\mathrm{qis}}, \mathcal{P}\right) \rightarrow$ $\operatorname{det}\left(\operatorname{Mod}(\Sigma)_{\text {iso }}, \mathcal{P}\right)$ is an equivalence of categories.

The inclusion of exact categories $\operatorname{PMod}(\Sigma) \rightarrow \operatorname{Mod}(\Sigma)$ induces a monoidal functor $\mathcal{V}(\operatorname{PMod}(\Sigma)) \rightarrow \mathcal{V}(\operatorname{Mod}(\Sigma))$, and the induced maps $\pi_{i} \mathcal{V}(\operatorname{PMod}(\Sigma)) \rightarrow$ $\pi_{i} \mathcal{V}(\operatorname{Mod}(\Sigma))$ for $i=0$ and 1 agree with the maps $K_{i}(\operatorname{PMod}(\Sigma)) \rightarrow K_{i}(\operatorname{Mod}(\Sigma))$ on algebraic $K$-groups (cf. $[\mathbf{6}, \S 4.11])$. Since $\Sigma$ is regular these maps are isomorphisms by Quillen's resolution theorem $[\mathbf{1 0}, \S 4$, Corollary 2], thus the monoidal functor $\mathcal{V}(\operatorname{PMod}(\Sigma)) \rightarrow \mathcal{V}(\operatorname{Mod}(\Sigma))$ is an equivalence of categories. Using the universal property of virtual objects one then easily deduces that $\operatorname{det}\left(\operatorname{Mod}(\Sigma)_{\text {iso }}, \mathcal{P}\right) \rightarrow$ $\operatorname{det}\left(\operatorname{PMod}(\Sigma)_{\text {iso }}, \mathcal{P}\right)$ is an equivalence of categories.

By (13) we can choose an extension of [·] $: \operatorname{PMod}(\Lambda)_{\text {iso }} \rightarrow \mathcal{V}(\Lambda)$ to a determinant functor $[\cdot]: \mathcal{C}^{b}(\operatorname{PMod}(\Lambda))_{\text {qis }} \rightarrow \mathcal{V}(\Lambda)$, and by Lemma 5.2 we can choose an extension of [.] $: \operatorname{PMod}(\Sigma)_{\text {iso }} \rightarrow \mathcal{V}(\Sigma)$ to a determinant functor [.] $: \mathcal{C}^{b}(\operatorname{Mod}(\Sigma))_{\text {qis }} \rightarrow \mathcal{V}(\Sigma)$. These extensions are unique up to isomorphism. Even though we use the same notation for all these determinant functors, it should always be clear from the context which one we mean. We will always work with the unique isomorphism in $\operatorname{det}\left(\mathcal{C}^{b}(\operatorname{PMod}(\Lambda))_{\text {qis }}, \mathcal{V}(\Sigma)\right)$ from the determinant functor $\mathcal{C}^{b}(\operatorname{PMod}(\Lambda))_{\text {qis }} \rightarrow$ $\mathcal{V}(\Lambda) \rightarrow \mathcal{V}(\Sigma)$ to the determinant functor $\mathcal{C}^{b}(\operatorname{PMod}(\Lambda))_{\text {qis }} \rightarrow \mathcal{C}^{b}(\operatorname{Mod}(\Sigma))_{\text {qis }} \rightarrow$ $\mathcal{V}(\Sigma)$ whose restriction to $\operatorname{PMod}(\Lambda)_{\text {iso }}$ is the isomorphism from $\operatorname{PMod}(\Lambda)_{\text {iso }} \rightarrow$ $\mathcal{V}(\Lambda) \rightarrow \mathcal{V}(\Sigma)$ to $\operatorname{PMod}(\Lambda)_{\text {iso }} \rightarrow \operatorname{PMod}(\Sigma)_{\text {iso }} \rightarrow \mathcal{V}(\Sigma)$ that we have fixed above. 
Remark 5.3. Obviously the definition of $\mathcal{V}(\Lambda, \Sigma)$ depends on the choice of the monoidal functor $\mathcal{V}(\Lambda) \rightarrow \mathcal{V}(\Sigma)$. Furthermore all our constructions in these categories (including the isomorphism in Lemma 5.1) depend on the choice of the determinant functors and isomorphisms of determinant functors. However one can check that the Euler characteristics defined below are independent of these choices. For semisimple $\Sigma$ this also follows from the results in $\S 6$.

Let $\mathcal{D}(\Lambda)$ be the derived category consisting of all complexes of $\Lambda$-modules. We remark that everything which follows also works if one imposes a boundedness condition on the complexes in $\mathcal{D}(\Lambda)$. A complex $P$ is called perfect if in $\mathcal{D}(\Lambda)$ it is isomorphic to a bounded complex of finitely generated projective $\Lambda$-modules. Equivalently, $P$ is perfect if there exists a quasi-isomorphism of complexes of $\Lambda$ modules $U \stackrel{\sim}{\longrightarrow} P$ with $U \in \mathcal{C}^{b}(\operatorname{PMod}(\Lambda))$. A perfect complex $P$ need not be bounded nor do we require the modules $P^{i}$ to be finitely generated. We denote the full subcategory of perfect complexes in $\mathcal{D}(\Lambda)$ by $\mathcal{D}^{\text {perf }}(\Lambda)$.

The categories $\mathcal{D}(\Sigma)$ and $\mathcal{D}^{\text {perf }}(\Sigma)$ are defined similarly. Since $\Sigma$ is noetherian, the category $\operatorname{Mod}(\Sigma)$ is abelian and $H(P)$ belongs to $\mathcal{C}^{b}(\operatorname{Mod}(\Sigma))$ for every perfect complex $P$ of $\Sigma$-modules.

Definition 5.4. Let $P \in \mathcal{D}^{\text {perf }}(\Lambda)$. We define the Euler characteristic $\chi_{\Lambda}(P) \in$ $K_{0}(\Lambda)$ to be the isomorphism class of the object $[U]$ in $\mathcal{V}(\Lambda)$ under the isomorphism $\pi_{0} \mathcal{V}(\Lambda) \cong K_{0}(\Lambda)$, where $U$ is a complex in $\mathcal{C}^{b}(\operatorname{PMod}(\Lambda))$ which is isomorphic to $P$ in $\mathcal{D}(\Lambda)$.

One easily verifies that $\chi_{\Lambda}(P)$ is well-defined. In addition, if $P \in \mathcal{C}^{b}(\operatorname{PMod}(\Lambda))$ then

$$
\chi_{\Lambda}(P)=\sum_{i}(-1)^{i}\left(P^{i}\right)
$$

in $K_{0}(\Lambda)$.

We write $H^{\mathrm{ev}}(P)$ and $H^{\text {od }}(P)$ for the direct sum of even and odd degree cohomology modules of a complex $P$.

Definition 5.5. Let $P \in \mathcal{D}^{\text {perf }}(\Lambda)$ and let $t: H^{\text {ev }}\left(P_{\Sigma}\right) \cong H^{\text {od }}\left(P_{\Sigma}\right)$ be an isomorphism of $\Sigma$-modules. We refer to $t$ as a 'trivialisation' of $P$ and to the pair $(P, t)$ as a 'trivialised complex'. We define the Euler characteristic $\chi_{\Lambda, \Sigma}(P, t)$ of the trivialised complex $(P, t)$ to be the element of $K_{0}(\Lambda, \Sigma)$ which is represented under the isomorphism $\pi_{0} \mathcal{V}(\Lambda, \Sigma) \cong K_{0}(\Lambda, \Sigma)$ of Lemma 5.1 by the object $([U], \lambda)$ in $\mathcal{V}(\Lambda, \Sigma)$, where $U$ and $\lambda$ are as follows. Choose a quasi-isomorphism of complexes of $\Lambda$-modules $a: U \stackrel{\sim}{\longrightarrow} P$ with $U \in \mathcal{C}^{b}(\operatorname{PMod}(\Lambda))$ and let $\lambda:[U]_{\Sigma} \rightarrow 1_{\mathcal{V}(\Sigma)}$ be the isomorphism in $\mathcal{V}(\Sigma)$ given by

$$
\begin{aligned}
& {[U]_{\Sigma} \cong\left[U_{\Sigma}\right] \stackrel{\eta_{U_{\Sigma}}}{\longrightarrow}\left[H\left(U_{\Sigma}\right)\right] \stackrel{\left[H\left(a_{\Sigma}\right)\right]}{\longrightarrow}\left[H\left(P_{\Sigma}\right)\right] \stackrel{\pi_{H\left(P_{\Sigma}\right)}}{\longrightarrow}\left[H^{\mathrm{ev}}\left(P_{\Sigma}\right)[0]\right] \otimes\left[H^{\mathrm{od}}\left(P_{\Sigma}\right)[1]\right] } \\
& \stackrel{[t] \otimes \mathrm{id}}{\longrightarrow}\left[H^{\mathrm{od}}\left(P_{\Sigma}\right)[0]\right] \otimes\left[H^{\mathrm{od}}\left(P_{\Sigma}\right)[1]\right] \stackrel{\mu_{H^{\mathrm{od}}\left(P_{\Sigma}\right)[0]}}{\longrightarrow} 1_{\mathcal{V}(\Sigma)} .
\end{aligned}
$$

Proposition 5.6. Let $P \in \mathcal{D}^{\text {perf }}(\Lambda)$ and let $t: H^{\mathrm{ev}}\left(P_{\Sigma}\right) \rightarrow H^{\text {od }}\left(P_{\Sigma}\right)$ be a trivialisation. Then the Euler characteristic $\chi_{\Lambda, \Sigma}(P, t) \in K_{0}(\Lambda, \Sigma)$ is well-defined and has the following properties. 
1. $\delta_{\Lambda, \Sigma}^{0}\left(\chi_{\Lambda, \Sigma}(P, t)\right)=\chi_{\Lambda}(P)$.

2. If $t^{\prime}: H^{\mathrm{ev}}\left(P_{\Sigma}\right) \rightarrow H^{\mathrm{od}}\left(P_{\Sigma}\right)$ is another trivialisation, then

$$
\chi_{\Lambda, \Sigma}\left(P, t^{\prime}\right)-\chi_{\Lambda, \Sigma}(P, t)=\delta_{\Lambda, \Sigma}^{1}\left(\left(H^{\text {od }}\left(P_{\Sigma}\right), t^{\prime} \circ t^{-1}\right)\right) .
$$

3. $\chi_{\Lambda, \Sigma}\left(P[1], t^{-1}\right)=-\chi_{\Lambda, \Sigma}(P, t)$.

Proof. If $a: U \stackrel{\sim}{\longrightarrow} P$ and $a^{\prime}: U^{\prime} \stackrel{\sim}{\longrightarrow} P$ are two quasi-isomorphisms, then there exists a quasi-isomorphism $b: U \stackrel{\sim}{\longrightarrow} U^{\prime}$ such that $a$ and $a^{\prime} \circ b$ are homotopic. From this the well-definedness easily follows.

1. Let $a: U \stackrel{\sim}{\longrightarrow} P$ and $\lambda$ be as in Definition 5.5. The functor $\mathcal{V}(\Lambda, \Sigma) \rightarrow \mathcal{V}(\Lambda)$ maps the isomorphism class of $([U], \lambda)$ in $\mathcal{V}(\Lambda, \Sigma)$ to the isomorphism class of $[U]$ in $\mathcal{V}(\Lambda)$. This shows $\delta_{\Lambda, \Sigma}^{1}\left(\chi_{\Lambda, \Sigma}(P, t)\right)=\chi_{\Lambda}(P)$.

2. We can assume that $P \in \mathcal{C}^{b}(\operatorname{PMod}(\Lambda))$. Let $\lambda_{t}, \lambda_{t^{\prime}}:[P]_{\Sigma} \rightarrow 1_{\mathcal{V}(\Sigma)}$ be the maps from Definition 5.5 for $t$ and $t^{\prime}$ respectively. Then one has $\lambda_{t^{\prime}}=\alpha \circ \lambda_{t}$ where $\alpha \in \operatorname{Aut}_{\mathcal{V}(\Sigma)}\left(1_{\mathcal{V}(\Sigma)}\right)$ is $\left[t^{\prime} \circ t^{-1}\right] \in \operatorname{Aut}_{\mathcal{V}(\Sigma)}\left(\left[H^{\text {od }}\left(P_{\Sigma}\right)[0]\right]\right)$ under the isomorphism $\operatorname{Aut}_{\mathcal{V}(\Sigma)}\left(1_{\mathcal{V}(\Sigma)}\right) \cong \operatorname{Aut}_{\mathcal{V}(\Sigma)}\left(\left[H^{\text {od }}\left(P_{\Sigma}\right)[0]\right]\right)$. This implies that $\left([P], \lambda_{t^{\prime}}\right)$ is isomorphic to $\left([P], \lambda_{t}\right) \otimes\left(1_{\mathcal{V}(\Lambda)}, \alpha\right)$ in $\mathcal{V}(\Lambda, \Sigma)$ which gives the result.

3. We can assume that $P \in \mathcal{C}^{b}(\operatorname{PMod}(\Lambda))$. The sum $\chi_{\Lambda, \Sigma}(P, t)+\chi_{\Lambda, \Sigma}\left(P[1], t^{-1}\right)$ is represented by the isomorphism class of $([P] \otimes[P[1]], \lambda \otimes \tilde{\lambda})$ in $\mathcal{V}(\Lambda, \Sigma)$, where $\lambda:[P]_{\Sigma} \rightarrow 1_{\mathcal{V}(\Sigma)}$ is associated to $t$ and $\tilde{\lambda}:[P[1]]_{\Sigma} \rightarrow 1_{\mathcal{V}(\Sigma)}$ is associated to $t^{-1}$. The following diagram

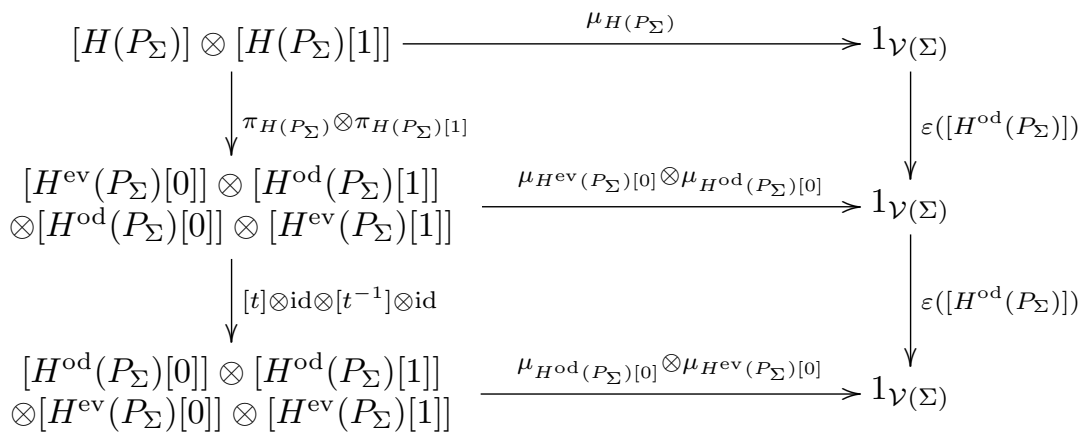

commutes in $\mathcal{V}(\Sigma)$ (this follows from Lemma 4.5 for the top square and from the definition of $\varepsilon$ for the bottom square). Together with Lemma 3.5 (applied to the complex $\left.P_{\Sigma}\right)$ this shows that $([P] \otimes[P[1]], \lambda \otimes \tilde{\lambda})$ is isomorphic to $\left(1_{\mathcal{V}(\Lambda)}, \mathrm{id}_{1_{\mathcal{V}(\Sigma)}}\right)$ in $\mathcal{V}(\Lambda, \Sigma)$ and therefore represents the zero element in $K_{0}(\Lambda, \Sigma)$.

We consider the following triangulation on $\mathcal{D}(\Lambda)$. A triangle

$$
P \stackrel{a}{\longrightarrow} Q \stackrel{b}{\longrightarrow} R \stackrel{c}{\longrightarrow} P[1]
$$

in $\mathcal{D}(\Lambda)$ is said to be 'distinguished' if it is isomorphic to a triangle of the form

$$
U \stackrel{e}{\longrightarrow} V \longrightarrow \operatorname{cone}(e) \longrightarrow U[1]
$$

where $e$ is a morphism of complexes and in each degree $i$ the map $V^{i} \rightarrow \operatorname{cone}(e)^{i}=$ $V^{i} \oplus U^{i+1}$ is the canonical inclusion, that is $v \mapsto(v, 0)$, and the map cone $(e)^{i}=V^{i} \oplus$ 
$U^{i+1} \rightarrow U^{i+1}$ is the negative of the canonical projection, that is $(v, u) \mapsto-u$. (The reader should be warned that some authors use the canonical projection instead of its negative which leads to a different triangulation on $\mathcal{D}(\Lambda)$ - see Remark 5.9.) One easily verifies that $\mathcal{D}^{\text {perf }}(\Lambda)$ is a triangulated subcategory of $\mathcal{D}(\Lambda)$.

Theorem 5.7. Let $P \stackrel{a}{\rightarrow} Q \stackrel{b}{\rightarrow} R \stackrel{c}{\rightarrow} P[1]$ be a distinguished triangle in $\mathcal{D}^{\text {perf }}(\Lambda)$. Let $t_{P}: H^{\mathrm{ev}}\left(P_{\Sigma}\right) \rightarrow H^{\mathrm{od}}\left(P_{\Sigma}\right), t_{Q}: H^{\mathrm{ev}}\left(Q_{\Sigma}\right) \rightarrow H^{\mathrm{od}}\left(Q_{\Sigma}\right)$ and $t_{R}: H^{\mathrm{ev}}\left(R_{\Sigma}\right) \rightarrow$ $H^{\mathrm{od}}\left(R_{\Sigma}\right)$ be trivialisations. If the following diagram

$$
\begin{aligned}
& {\left[H^{\mathrm{ev}} Q_{\Sigma}\right] \otimes\left[\operatorname{ker}\left(H^{\mathrm{ev}} a_{\Sigma}\right)\right] \otimes\left[\operatorname{ker}\left(H^{\mathrm{od}} a_{\Sigma}\right)\right] \longrightarrow\left[H^{\mathrm{ev}} P_{\Sigma}\right] \otimes\left[H^{\mathrm{ev}} R_{\Sigma}\right]} \\
& \downarrow\left[t_{Q}\right] \otimes \mathrm{id} \otimes[-\mathrm{id}] \quad \downarrow\left[t_{P}\right] \otimes\left[t_{R}\right] \\
& {\left[H^{\mathrm{od}} Q_{\Sigma}\right] \otimes\left[\operatorname{ker}\left(H^{\mathrm{ev}} a_{\Sigma}\right)\right] \otimes\left[\operatorname{ker}\left(H^{\mathrm{od}} a_{\Sigma}\right)\right] \longrightarrow\left[H^{\mathrm{od}} P_{\Sigma}\right] \otimes\left[H^{\mathrm{od}} R_{\Sigma}\right]}
\end{aligned}
$$

commutes in $\mathcal{V}(\Sigma)$, then

$$
\chi_{\Lambda, \Sigma}\left(Q, t_{Q}\right)=\chi_{\Lambda, \Sigma}\left(P, t_{P}\right)+\chi_{\Lambda, \Sigma}\left(R, t_{R}\right)
$$

in $K_{0}(\Lambda, \Sigma)$. The horizontal arrows in diagram (15) are induced by the exact sequences

$$
\begin{aligned}
& 0 \rightarrow \operatorname{ker}\left(H^{\mathrm{ev}} a_{\Sigma}\right) \rightarrow H^{\mathrm{ev}} P_{\Sigma} \rightarrow H^{\mathrm{ev}} Q_{\Sigma} \rightarrow H^{\mathrm{ev}} R_{\Sigma} \rightarrow \operatorname{ker}\left(H^{\mathrm{od}} a_{\Sigma}\right) \rightarrow 0 \\
& 0 \rightarrow \operatorname{ker}\left(H^{\mathrm{od}} a_{\Sigma}\right) \rightarrow H^{\mathrm{od}} P_{\Sigma} \rightarrow H^{\mathrm{od}} Q_{\Sigma} \rightarrow H^{\mathrm{od}} R_{\Sigma} \rightarrow \operatorname{ker}\left(H^{\mathrm{ev}} a_{\Sigma}\right) \rightarrow 0
\end{aligned}
$$

which are obtained from the long exact cohomology sequence of the distinguished triangle $P_{\Sigma} \stackrel{a_{\Sigma}}{\longrightarrow} Q_{\Sigma} \stackrel{b_{\Sigma}}{\longrightarrow} R_{\Sigma} \stackrel{c_{\Sigma}}{\longrightarrow} P_{\Sigma}[1]$.

Proof. The following lemma reduces the proof of Theorem 5.7 to the case of a short exact sequence.

Lemma 5.8. For every distinguished triangle $P \stackrel{a}{\rightarrow} Q \stackrel{b}{\rightarrow} R \stackrel{c}{\rightarrow} P[1]$ in $\mathcal{D}^{\text {perf }}(\Lambda)$ there exists a short exact sequence $0 \rightarrow U \rightarrow V \rightarrow W \rightarrow 0$ in $\mathcal{C}^{b}(\operatorname{PMod}(\Lambda))$ and quasi-isomorphisms of complexes of $\Lambda$-modules $U \stackrel{\sim}{\longrightarrow} P, V \stackrel{\sim}{\longrightarrow} Q, W \stackrel{\sim}{\longrightarrow} R$ such that the induced diagram of cohomology sequences

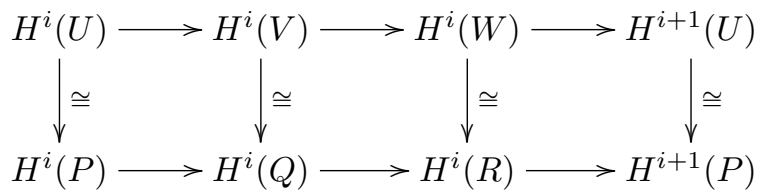

is commutative.

Proof. Choose quasi-isomorphisms $U \stackrel{\sim}{\longrightarrow} P$ and $X \stackrel{\sim}{\longrightarrow} R[-1]$ with $U$ and $X$ in $\mathcal{C}^{b}(\operatorname{PMod}(\Lambda))$. Let $e: X \rightarrow U$ be a map of complexes such that the left hand square in the following diagram in $\mathcal{D}(\Lambda)$ commutes.

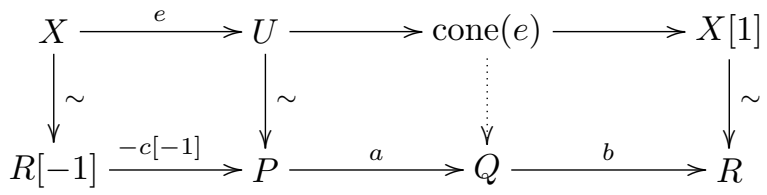


The dotted arrow making everything commutative exists by the axioms of a triangulated category. In fact, since cone $(e)$ is a bounded complex of finitely generated projective $\Lambda$-modules we can assume that the dotted arrow comes from a map of complexes of $\Lambda$-modules cone $(e) \rightarrow Q$ which then must be a quasi-isomorphism. Shifting back we see that the given distinguished triangle is isomorphic to the distinguished triangle

$$
U \longrightarrow \operatorname{cone}(e) \longrightarrow X[1] \stackrel{-e[1]}{\longrightarrow} U[1]
$$

and therefore has an isomorphic cohomology sequence. An easy computation shows that the connecting homomorphism in the long exact cohomology sequence coming from the short exact sequence $0 \rightarrow U \rightarrow \operatorname{cone}(e) \rightarrow X[1] \rightarrow 0$ is induced by $-e$. Therefore the cohomology sequences of the distinguished triangle and of the short exact sequence are isomorphic.

We continue with the proof of Theorem 5.7. By Lemma 5.8 and the definition of the Euler characteristics, it suffices to prove the result for a short exact sequence $\Delta: 0 \rightarrow P \stackrel{a}{\rightarrow} Q \stackrel{b}{\rightarrow} R \rightarrow 0$ in $\mathcal{C}^{b}(\operatorname{PMod}(\Lambda))$. In this case we will show that the following diagram



commutes in $\mathcal{V}(\Sigma)$, where $\lambda_{Q}, \lambda_{P}$ and $\lambda_{R}$ are the maps from Definition 5.5. Note that the commutativity of this diagram implies that $\left([Q], \lambda_{Q}\right)$ and $\left([P], \lambda_{P}\right) \otimes\left([R], \lambda_{R}\right)$ are isomorphic in $\mathcal{V}(\Lambda, \Sigma)$, and hence ensures that $\chi_{\Lambda, \Sigma}\left(Q, t_{Q}\right)=\chi_{\Lambda, \Sigma}\left(P, t_{P}\right)+$ $\chi_{\Lambda, \Sigma}\left(R, t_{R}\right)$, as required.

For typographical simplicity, we will omit the subscript $\Sigma$ on all complexes and maps in the rest of this proof. The key observation is the existence of a commutative diagram




In the diagram the vertical maps are as indicated, the map $\alpha_{1}$ is obtained by applying $\mu_{\operatorname{ker}(H a)}^{-1}$ and the maps $\alpha_{2}$ and $\alpha_{3}$ are both obtained by applying $\mu_{\operatorname{ker}\left(H^{\mathrm{ev}} a\right)[0]}^{-1}$

and $\mu_{\operatorname{ker}\left(H^{\text {od }} a\right)[0]}^{-1}$. Furthermore $\varepsilon$ denotes the map $\varepsilon\left(\left[\operatorname{ker}\left(H^{\text {od }} a\right)\right]\right)$. The map $\beta_{1}$ is induced by the cohomology sequence

$$
0 \longrightarrow \operatorname{ker}(H a) \longrightarrow H P \longrightarrow H Q \longrightarrow H R \longrightarrow \operatorname{ker}(H a)[1] \longrightarrow 0,
$$

and the maps $\beta_{2}$ and $\beta_{3}$ are induced by the cohomology sequences (16) and (17). The top left square is commutative by Lemma 4.5 and the top right square by the compatibility of $\pi$ with respect to exact sequences. For the middle left square commutativity follows since [-id] acting on $\left[\operatorname{ker}\left(H^{\text {od }} a\right)[0]\right]$ agrees with $\varepsilon\left(\left[\operatorname{ker}\left(H^{\text {od }} a\right)\right]\right)$ and for the middle right square it follows by tensoring diagram (15) with

$$
\left[H^{\text {od }} Q[1]\right] \otimes\left[\operatorname{ker}\left(H^{\text {od }} a\right)[1]\right] \otimes\left[\operatorname{ker}\left(H^{\mathrm{ev}} a\right)[1]\right] \longrightarrow\left[H^{\text {od }} P[1]\right] \otimes\left[H^{\text {od }} R[1]\right]
$$

(induced by the exact sequence (17)). Finally for the bottom left square commutativity is obvious and for the bottom right square it follows from the compatibility of $\mu$ with exact sequences.

Together with Theorem 3.3 this implies that diagram (18) commutes and hence completes the proof of Theorem 5.7.

Remark 5.9. An alternative triangulation on $\mathcal{D}(\Lambda)$ consists of triangles isomorphic to those of the form

$$
U \stackrel{e}{\longrightarrow} V \longrightarrow \operatorname{cone}(e) \longrightarrow U[1]
$$

where $V \rightarrow$ cone $(e)$ is the canonical inclusion and cone $(e) \rightarrow U[1]$ is the canonical projection. A triangle $P \stackrel{a}{\rightarrow} Q \stackrel{b}{\rightarrow} R \stackrel{c}{\rightarrow} P[1]$ is distinguished for this triangulation if and only if $P \stackrel{a}{\rightarrow} Q \stackrel{b}{\rightarrow} R \stackrel{-c}{\longrightarrow} P[1]$ is distinguished for the previous triangulation. Using this observation one can apply Theorem 5.7 to obtain an analogous additivity criterion for this alternative triangulation.

\section{Euler characteristics in the semisimple case}

As in $\S 5$ we consider a ring homomorphism $\Lambda \rightarrow \Sigma$ such that $\Sigma$ is flat as right $\Lambda$-module, but in this section we assume that $\Sigma$ is semisimple. We fix an object $P$ of $\mathcal{D}^{\text {perf }}(\Lambda)$ and let $t: H^{\text {ev }}\left(P_{\Sigma}\right) \stackrel{\cong}{\longrightarrow} H^{\text {od }}\left(P_{\Sigma}\right)$ be a trivialisation. In $[3]$ the second named author gave an explicit construction of a 'refined Euler characteristic' of such a complex $P$ and isomorphism $t^{-1}: H^{\text {od }}\left(P_{\Sigma}\right) \rightarrow H^{\mathrm{ev}}\left(P_{\Sigma}\right)$ (in a slightly more restrictive setting - see Remark 6.1 below). This refined Euler characteristic again lies in the relative algebraic $K$-group $K_{0}(\Lambda, \Sigma)$ and we will denote it by $\chi^{\text {old }}\left(P, t^{-1}\right)$.

If $P$ belongs to $\mathcal{C}^{b}(\operatorname{PMod}(\Lambda))$, then the refined Euler characteristic is defined by setting $\chi^{\text {old }}\left(P, t^{-1}\right)=\left(P^{\text {od }}, g, P^{\text {ev }}\right)$ where $g$ is the composite isomorphism

$$
P_{\Sigma}^{\text {od }} \stackrel{\cong}{\longrightarrow} B^{\text {all }}\left(P_{\Sigma}\right) \oplus H^{\text {od }}\left(P_{\Sigma}\right) \stackrel{\text { id } \oplus t^{-1}}{\longrightarrow} B^{\text {all }}\left(P_{\Sigma}\right) \oplus H^{\text {ev }}\left(P_{\Sigma}\right) \stackrel{\cong}{\longrightarrow} P_{\Sigma}^{\text {ev }} .
$$

Here $B^{\text {all }}\left(P_{\Sigma}\right)=\bigoplus_{i \in \mathbb{Z}} B^{i}\left(P_{\Sigma}\right)=B^{\mathrm{ev}}\left(P_{\Sigma}\right) \oplus B^{\text {od }}\left(P_{\Sigma}\right)$. The first and third isomorphism depend on the choice of splittings of the tautological exact sequences $0 \rightarrow Z^{i}\left(P_{\Sigma}\right) \rightarrow P_{\Sigma}^{i} \rightarrow B^{i+1}\left(P_{\Sigma}\right) \rightarrow 0$ and $0 \rightarrow B^{i}\left(P_{\Sigma}\right) \rightarrow Z^{i}\left(P_{\Sigma}\right) \rightarrow H^{i}\left(P_{\Sigma}\right) \rightarrow 0$, 
but it can be shown that $\chi^{\text {old }}\left(P, t^{-1}\right)$ is independent of all such choices. For a general complex $P \in \mathcal{D}^{\text {perf }}(\Lambda)$ one first chooses a quasi-isomorphism of complexes of $\Lambda$-modules $U \stackrel{\sim}{\longrightarrow} P$ with $U \in \mathcal{C}^{b}(\operatorname{PMod}(\Lambda))$ and then applies the above construction to $U$ and the induced isomorphism $H^{\mathrm{ev}}\left(U_{\Sigma}\right) \rightarrow H^{\text {od }}\left(U_{\Sigma}\right)$.

Remark 6.1. The situation considered in $[\mathbf{3}]$ is as follows. Let $R$ be a Dedekind domain of characteristic 0 with field of fractions $F$ and $E$ an extension field of $F$. Let $A$ be a finite dimensional semisimple $F$-algebra and $\mathcal{A}$ an $R$-order in $A$. Then $\Lambda=\mathcal{A}$ and $\Sigma=E \otimes_{F} A$ satisfies the assumptions considered above. If $P \in \mathcal{D}^{\text {perf }}(\mathcal{A})$ and $t: H^{\mathrm{ev}}\left(E \otimes_{R} P\right) \rightarrow H^{\mathrm{od}}\left(E \otimes_{R} P\right)$ is a trivialisation, then the refined Euler characteristic denoted by $\chi_{\mathcal{A}}\left(P, t^{-1}\right) \in K_{0}\left(\mathcal{A}, E \otimes_{F} A\right)$ in $[\mathbf{3}]$ is the element we denoted by $\chi^{\text {old }}\left(P, t^{-1}\right)$.

Theorem 6.2. If $P$ is an object of $\mathcal{D}^{\text {perf }}(\Lambda)$ and $t: H^{\mathrm{ev}}\left(P_{\Sigma}\right) \stackrel{\cong}{\rightrightarrows} H^{\text {od }}\left(P_{\Sigma}\right) a$ trivialisation, then

$$
-\chi^{\mathrm{old}}\left(P, t^{-1}\right)=\chi_{\Lambda, \Sigma}(P, t)+\delta_{\Lambda, \Sigma}^{1}\left(\left(B^{\text {od }}\left(P_{\Sigma}\right),-\mathrm{id}\right)\right)
$$

in $K_{0}(\Lambda, \Sigma)$.

Proof. By Lemma 6.3 below, the term $\delta_{\Lambda, \Sigma}^{1}\left(\left(B^{\text {od }}\left(P_{\Sigma}\right),-\right.\right.$ id $\left.)\right)$ does not change if we replace $P$ by a quasi-isomorphic complex. Without loss of generality we can therefore assume that $P \in \mathcal{C}^{b}(\operatorname{PMod}(\Lambda))$. One has $-\chi^{\text {old }}\left(P, t^{-1}\right)=\left(P^{\mathrm{ev}}, g^{-1}, P^{\text {od }}\right)$ and by Lemma 5.1 and the identification $[P] \stackrel{\pi_{P}}{\longrightarrow}\left[P^{\mathrm{ev}}[0]\right] \otimes\left[P^{\mathrm{od}}[1]\right] \rightarrow\left[P^{\mathrm{ev}}\right] \otimes\left[P^{\mathrm{od}}\right]^{-1}$ this element is represented by the isomorphism class of

$$
\left([P], \mu_{P_{\Sigma}^{\text {od }}[0]} \circ\left(\left[g^{-1}\right] \otimes \mathrm{id}\right) \circ \pi_{P_{\Sigma}}\right)
$$

in $\mathcal{V}(\Lambda, \Sigma)$. On the other hand $\chi_{\Lambda, \Sigma}(P, t)$ is represented by the isomorphism class of $([P], \lambda)$ in $\mathcal{V}(\Lambda, \Sigma)$, where $\lambda:\left[P_{\Sigma}\right] \rightarrow 1_{\mathcal{V}(\Sigma)}$ is the map from Definition 5.5. We will show that

$$
\lambda=\varepsilon\left(\left[B^{\text {od }} P_{\Sigma}\right]\right) \circ \mu_{P_{\Sigma}^{\text {od }}[0]} \circ\left(\left[g^{-1}\right] \otimes \mathrm{id}\right) \circ \pi_{P_{\Sigma}}
$$

which then immediately implies the claimed equality in $K_{0}(\Lambda, \Sigma)$.

To simplify the notation we omit the subscript $\Sigma$ for the rest of this proof. Note that since one has $\lambda=\mu \circ([t] \otimes$ id $) \circ \pi \circ \beta_{1} \circ \alpha_{1}$ in the notation of the diagram below, the commutativity of the outer square of that diagram will imply the required equation (19). 




The vertical maps in this diagram are as indicated. The map $\alpha_{1}$ is induced by the filtration $0 \subseteq B P \subseteq Z P \subseteq P$ with quotients $B P, H P$ and $B P[1]$, and the maps $\alpha_{2}$ and $\alpha_{3}$ are induced by the filtration $0 \subseteq B^{\mathrm{ev}} P \subseteq Z^{\mathrm{ev}} P \subseteq P^{\mathrm{ev}}$ with quotients $B^{\text {ev }} P, H^{\text {ev }} P$ and $B^{\text {od }} P$, and the filtration $0 \subseteq B^{\text {od }} P \subseteq Z^{\text {od }} P \subseteq P^{\text {od }}$ with quotients $B^{\text {od }} P, H^{\text {od }} P$ and $B^{\text {ev }} P$. The commutativity of all squares on the left follows from the compatibility of $\pi$ and $\mu$ with respect to filtrations and from the definition of $g$. The map $\beta_{1}$ is obtained by applying $\mu_{B P}$ and the maps $\beta_{2}$ and $\beta_{3}$ are obtained by applying $\mu_{B^{\mathrm{ev}} P[0]}$ and $\mu_{B^{\text {od }} P[0]}$. The commutativity of the upper right hand square is Lemma 4.5. The other two squares on the right hand side are obviously commutative.

When applying Theorem 6.2 it is useful to note that $\delta_{\Lambda, \Sigma}^{1}\left(\left(B^{\text {od }}\left(P_{\Sigma}\right),-\right.\right.$ id $\left.)\right)$ can be expressed purely in terms of the cohomology of $P_{\Sigma}$ as follows.

Lemma 6.3. One has

$$
\begin{aligned}
\delta_{\Lambda, \Sigma}^{1}\left(\left(B^{\mathrm{od}}\left(P_{\Sigma}\right),-\mathrm{id}\right)\right) & =\delta_{\Lambda, \Sigma}^{1}\left(\left(\bigoplus_{i \equiv 1,2 \bmod 4} H^{i}\left(P_{\Sigma}\right),-\mathrm{id}\right)\right) \\
& =\delta_{\Lambda, \Sigma}^{1}\left(\left(\bigoplus_{i \equiv 0,3 \bmod 4} H^{i}\left(P_{\Sigma}\right),-\mathrm{id}\right)\right) .
\end{aligned}
$$

Proof. Using the tautological exact sequences $0 \rightarrow Z^{i} P_{\Sigma} \rightarrow P_{\Sigma}^{i} \rightarrow B^{i+1} P_{\Sigma} \rightarrow 0$ and $0 \rightarrow B^{i} P_{\Sigma} \rightarrow Z^{i} P_{\Sigma} \rightarrow H^{i} P_{\Sigma} \rightarrow 0$ and the observation that $\delta_{\Lambda, \Sigma}^{1}\left(\left(P_{\Sigma}^{i},-\mathrm{id}\right)\right)=$ $0 \in K_{0}(\Lambda, \Sigma)$, one finds that

$$
\begin{aligned}
& \delta_{\Lambda, \Sigma}^{1}\left(\left(B^{i} P_{\Sigma},-\mathrm{id}\right)\right)+\delta_{\Lambda, \Sigma}^{1}\left(\left(B^{i+2} P_{\Sigma},-\mathrm{id}\right)\right) \\
& \quad=\delta_{\Lambda, \Sigma}^{1}\left(\left(H^{i} P_{\Sigma},-\mathrm{id}\right)\right)+\delta_{\Lambda, \Sigma}^{1}\left(\left(H^{i+1} P_{\Sigma},-\mathrm{id}\right)\right) .
\end{aligned}
$$

The two equalities in the lemma follow by adding over all $i \equiv 1 \bmod 4$ and all $i \equiv 3 \bmod 4$ respectively.

Remark 6.4. In arithmetic applications one often deals with complexes that are acyclic outside two consecutive degrees. With this in mind, we note that Lemma 
6.3 implies that if $P_{\Sigma}$ is acyclic outside degrees 0 and 1 , then

$$
\delta_{\Lambda, \Sigma}^{1}\left(\left(B^{\text {od }} P_{\Sigma},-\mathrm{id}\right)\right)=\delta_{\Lambda, \Sigma}^{1}\left(\left(H^{0} P_{\Sigma},-\mathrm{id}\right)\right)=\delta_{\Lambda, \Sigma}^{1}\left(\left(H^{1} P_{\Sigma},-\mathrm{id}\right)\right),
$$

whilst if $P_{\Sigma}$ is acyclic outside degrees 1 and 2, then Lemma 6.3 implies that

$$
\delta_{\Lambda, \Sigma}^{1}\left(\left(B^{\text {od }} P_{\Sigma},-\mathrm{id}\right)\right)=0 .
$$

Remark 6.5. The result of Theorem 6.2 implies that the final sentence of $[\mathbf{4}$, Remark $4]$ is incorrect as stated. Specifically, whilst it is clear that the sentence in question is correct if one replaces the term ' $\chi_{\mathfrak{A}}\left(Y, \psi^{-1}\right)$ ' by (in our notation) the inverse of the element $\chi_{\mathfrak{A}, A_{F}}(Y, \psi)$, Theorem 6.2 shows that these terms do not in general coincide. For a similar reason, the equality of $[\mathbf{3},(3.1)]$ is valid in general only if the morphism ' $\tau_{\text {Triv }}$ ' is replaced by ' $\epsilon_{C} \cdot \cdot \tau_{\text {Triv' }}$ ' where $\epsilon_{C} \cdot \in A^{\times}$is equal to the product of the determinants (over $A$ ) of the action of -id on certain cohomology modules $H^{i}\left(C^{\cdot}\right)$ (but to be more specific in this regard one would need to be precise about certain sign conventions used in $[\mathbf{3}])$. This change to $[\mathbf{3},(3.1)]$ in turn necessitates a corresponding change in the statement of [3, Theorem 3.1]. The second named author would like to apologise to the reader for these errors.

We finish this paper with a corollary of Theorem 5.7 which gives a more explicit version of the additivity criterion under the assumption that $\Sigma$ is semisimple. We recall that in this case each automorphism $\alpha$ of a finitely generated $\Sigma$-module gives rise to a 'reduced norm' element $\operatorname{nr}_{\Sigma}(\alpha)$ in the multiplicative group $\zeta(\Sigma)^{\times}$of the centre of $\Sigma$ and that the association $\alpha \mapsto \operatorname{nr}_{\Sigma}(\alpha)$ induces a well-defined group homomorphism $K_{1}(\Sigma) \rightarrow \zeta(\Sigma)^{\times}$.

Corollary 6.6. Assume that $\Sigma$ is semisimple and that the homomorphism $K_{1}(\Sigma) \rightarrow$ $\zeta(\Sigma)^{\times}$induced by the reduced norm $\mathrm{nr}_{\Sigma}$ is injective. Let $P \stackrel{a}{\rightarrow} Q \stackrel{b}{\rightarrow} R \stackrel{c}{\rightarrow} P[1]$ and $t_{P}, t_{Q}, t_{R}$ be as in Theorem 5.7. Let

$$
\begin{aligned}
& s^{\text {ev }}: H^{\text {ev }} Q_{\Sigma} \oplus \operatorname{ker}\left(H^{\mathrm{ev}} a_{\Sigma}\right) \oplus \operatorname{ker}\left(H^{\text {od }} a_{\Sigma}\right) \longrightarrow H^{\text {ev }} P_{\Sigma} \oplus H^{\text {ev }} R_{\Sigma}, \\
& s^{\text {od }}: H^{\text {od }} Q_{\Sigma} \oplus \operatorname{ker}\left(H^{\text {ev }} a_{\Sigma}\right) \oplus \operatorname{ker}\left(H^{\text {od }} a_{\Sigma}\right) \longrightarrow H^{\text {od }} P_{\Sigma} \oplus H^{\text {od }} R_{\Sigma}
\end{aligned}
$$

be isomorphisms induced by any choice of splittings of the exact sequences (16) and (17) respectively. If in $\zeta(\Sigma)^{\times}$one has

$$
\operatorname{nr}_{\Sigma}\left(\left(t_{Q}^{-1} \oplus \mathrm{id} \oplus(-\mathrm{id})\right) \circ\left(s^{\mathrm{od}}\right)^{-1} \circ\left(t_{P} \oplus t_{R}\right) \circ s^{\mathrm{ev}}\right)=1,
$$

then in $K_{0}(\Lambda, \Sigma)$ one has

$$
\chi_{\Lambda, \Sigma}\left(Q, t_{Q}\right)=\chi_{\Lambda, \Sigma}\left(P, t_{P}\right)+\chi_{\Lambda, \Sigma}\left(R, t_{R}\right) .
$$

Proof. For brevity we set $\alpha=\left(t_{Q}^{-1} \oplus \mathrm{id} \oplus(-\mathrm{id})\right) \circ\left(s^{\mathrm{od}}\right)^{-1} \circ\left(t_{P} \oplus t_{R}\right) \circ s^{\mathrm{ev}}$ and $W=H^{\mathrm{ev}} Q_{\Sigma} \oplus \operatorname{ker}\left(H^{\mathrm{ev}} a_{\Sigma}\right) \oplus \operatorname{ker}\left(H^{\mathrm{od}} a_{\Sigma}\right)$. The assumptions of Corollary 6.6 imply that $[\alpha]=\operatorname{id}_{[W]}$ in $\operatorname{Aut}_{\mathcal{V}(\Sigma)}([W])$ because $\operatorname{nr}_{\Sigma}(\alpha)$ is the image of $[\alpha]$ under the composite homomorphism Aut $\operatorname{\mathcal {V}}(\Sigma)_{(}([W]) \cong \pi_{1}(\mathcal{V}(\Sigma)) \cong K_{1}(\Sigma) \rightarrow \zeta(\Sigma)^{\times}$, where the first two maps are the canonical isomorphisms and the last arrow denotes the homomorphism induced by $\mathrm{nr}_{\Sigma}$. But $[\alpha]=\mathrm{id}_{[W]}$ is equivalent to the commutativity of diagram (15) and so the claimed result follows directly from Theorem 5.7. 
The reduced norm map $K_{1}(\Sigma) \rightarrow \zeta(\Sigma)^{\times}$can fail to be injective even in natural arithmetic contexts (for example, it is known to have non-trivial kernel if $\Sigma$ is the total quotient ring of the Iwasawa algebra of a semidirect product of the form $\left.\mathbb{Z}_{p} \rtimes \mathbb{Z}_{p}\right)$. However, if $E$ is a finite extension of either $\mathbb{Q}$ or $\mathbb{Q}_{p}$ for any prime $p$, then the map is always injective in the setting of Remark 6.1 (cf. [5, (45.3)]). In addition, the criterion of Corollary 6.6 is straightforward to check in the case $H c_{\Sigma}=0$ (so that $\left.\operatorname{ker}\left(H^{\mathrm{ev}} a_{\Sigma}\right)=\operatorname{ker}\left(H^{\mathrm{od}} a_{\Sigma}\right)=0\right)$. In particular, in this way one can recover the result of [3, Theorem 2.8] as an easy special case of Corollary 6.6.

\section{References}

[1] W. Bley, D. Burns, Equivariant epsilon constants, discriminants and étale cohomology, Proc. London Math. Soc. 87 (2003), 545-590.

[2] D. Burns, Equivariant Tamagawa numbers and Galois module theory I, Compositio Math. 129 (2001), 203-237.

[3] D. Burns, Equivariant Whitehead torsion and refined Euler characteristics, in: Number theory, pp. 35-59, CRM Proc. and Lecture Notes 36, Amer. Math. Soc., Providence, 2004.

[4] D. Burns, M. Flach, Tamagawa numbers for motives with (noncommutative) coefficients, Doc. Math. 6 (2001), 501-570.

[5] C. W. Curtis, I. Reiner, Methods of Representation Theory, Vol. II, John Wiley \& Sons, New York, 1987.

[6] P. Deligne, Le déterminant de la cohomologie, in: Current trends in arithmetical algebraic geometry (Arcata, CA, 1985), pp. 93-177, Contemp. Math. 67, Amer. Math. Soc., Providence, 1987.

[7] T. Fukaya, K. Kato, A formulation of conjectures on p-adic zeta functions in non-commutative Iwasawa theory, to appear in Proc. St. Petersburg Math. Soc. 11 (2005).

[8] F. Knudsen, Determinant functors on exact categories and their extensions to categories of bounded complexes, Michigan Math. J. 50 (2002), 407-444.

[9] F. Knudsen, D. Mumford, The projectivity of the moduli space of stable curves, I: preliminaries on "det" and "Div", Math. Scand. 39 (1976), 1955 .

[10] D. Quillen, Higher algebraic K-theory: I, in: Algebraic K-theory, I: Higher K-theories (Seattle, Wash., 1972), pp. 85-147, Lecture Notes in Math. 341, Springer, Berlin, 1973. 
[11] N. Saavedra Rivano, Catégories Tannakiennes, Lecture Notes in Math. 265, Springer, Berlin, 1972.

[12] R. G. Swan, Algebraic K-theory, Lecture Notes in Math. 76, Springer, Berlin, 1968.

This article is available at http://intlpress.com/HHA/v7/n3/a2/

Manuel Breuning manuel.breuning@maths.nottingham.ac.uk

School of Mathematical Sciences

University of Nottingham

University Park

Nottingham NG7 2RD

United Kingdom

David Burns david.burns@kcl.ac.uk

Department of Mathematics

King's College London

Strand

London WC2R 2LS

United Kingdom 